\title{
CHEREDNIK AND HECKE ALGEBRAS OF VARIETIES WITH A FINITE GROUP ACTION
}

\author{
PAVEL ETINGOF
}

\section{IntroduCtion}

This paper is an expanded and updated version of the unpublished 2004 preprint Et1. It includes a more detailed description of the basics of the theory of Cherednik and Hecke algebras of varieties started in [Et1, as well as a new Section 4, which reviews the developments in this theory since 2004 with references to the relevant literature.

Let $\mathfrak{h}$ be a finite dimensional complex vector space, and $G$ be a finite subgroup of $G L(\mathfrak{h})$. To this data one can attach a family of algebras $H_{t, c}(\mathfrak{h}, G)$, called the rational Cherednik algebras (see [EG]); for $t=1$ it provides the universal deformation of $G \ltimes D(\mathfrak{h})$ (where $D(\mathfrak{h})$ is the algebra of differential operators on $\mathfrak{h}$ ). These algebras are generated by $G, \mathfrak{h}, \mathfrak{h}^{*}$ with certain commutation relations, and are parametrized by pairs $(t, c)$, where $t$ is a complex number, and $c$ is a conjugation invariant function on the set of complex reflections in $G$. They have a rich representation theory and deep connections with combinatorics (Macdonald theory, $n$ ! conjecture) and algebraic geometry (Hilbert schemes, resolutions of symplectic quotient singularities).

The purpose of this paper is to introduce "global" analogues of rational Cherednik algebras, attached to any smooth complex algebraic variety $X$ with an action of a finite group $G$; the usual rational Cherednik algebras are recovered in the case when $X$ is a vector space and $G$ acts linearly.

More specifically, let $G$ be a finite group of automorphisms of $X$, and let $S$ be the set of pairs $(Y, g)$, where $g \in G$, and $Y$ is a connected component of the set $X^{g}$ of $g$-fixed points in $X$ which has codimension 1 in $X$ (we will call such a component a reflection hypersurface). Suppose that $X$ is affine. Then we define (in Section 2 of the paper) a family of algebras $H_{t, c, \omega}(X, G)$, where $t \in \mathbb{C}, c$ is a $G$-invariant function on $S$, and $\omega$ is a $G$-invariant closed 2 -form on $X$. This family for $t=1$ provides a universal deformation of the algebra $H_{1,0,0}(X, G)=G \ltimes D(X)$, where $D(X)$ is the algebra of differential operators on $X$ (assuming that $\omega$ runs through a space of forms bijectively representing $\left.H^{2}(X, \mathbb{C})^{G}\right)$.

If $X$ is not affine, then we define a sheaf of algebras $H_{t, c, \omega, X, G}$ rather than a single algebra. In this case the parameters are the same, except that $\omega$ runs over a space representing classes of $G$-equivariant twisted differential operator (tdo) algebras on $X$ (see $\mathrm{BB}$, Section 2).

We find that much of the theory of rational Cherednik algebras survives in the global case. In particular, one can define the spherical subalgebra, which is both commutative and isomorphic to the center of the Cherednik algebra in the case $t=0$. The spectrum of this algebra is "the Calogero-Moser space" of $X$, which is a global version of the similar space defined in $[\mathrm{EG}$. This includes, in particular, 
Calogero-Moser spaces attached to symmetric powers of algebraic curves. One can also define the global analog of the theory of quasiinvariants which was worked out in [FV, EG, BEG] and references therein. These results can be generalized to the case when $X$ is a complex analytic variety.

In Section 3, we discuss an application of the theory of global Cherednik algebras for complex analytic varieties. Namely, for an analytic $G$-variety $X$ we define its Hecke algebra, which is a certain explicitly defined formal deformation of the group algebra of the orbifold fundamental group of $X / G$. We show that if $\pi_{2}(X) \otimes \mathbb{Q}=0$ (a condition that cannot be removed), then the Hecke algebra is a flat deformation. This includes usual, affine, and double affine Hecke algebras for Weyl groups, as well as Hecke algebras for complex reflection groups. The proof is based on showing that the regular representation of the orbifold fundamental group can be deformed to a representation of the Hecke algebra. The required deformation is constructed by applying the KZ functor to a module over the global Cherednik algebra.

In Section 4 we review the developments in the theory of Cherednik and Hecke algebras of varieties with a finite group action since their introduction in the preprint Et1, and give the corresponding references. This section shows how these algebras fit into a bigger representation-theoretic, deformation-theoretic, and geometric picture.

Acknowledgements. This paper is dedicated to the the 80-th anniversary of V. I. Arnold. His books, courses, and his approach to mathematics in general have profoundly influenced me since my youth and will continue to do so in the future.

I am very grateful to A. Braverman for many explanations about D-modules, in particular for proofs of Propositions 2.1 and 2.3. I thank V. Ginzburg and A. Okounkov for useful discussions, and D. Thompson for comments on the draft of this paper. My work was partially supported by the NSF grants DMS-9988796, DMS-1502244, and the CRDF grant RM1-2545-MO-03.

\section{The Cherednik algebra of a $G$-VAriety}

2.1. Basic results about $D$-modules. In this subsection we will prove a few basic results about D-modules, to be used below. We refer the reader to the books Bo, HTT for basics on D-modules.

Throughout the paper, we work over $\mathbb{C}$. Let $X$ be a smooth algebraic variety. Let $\mathcal{O}_{X}$ be the structure sheaf of $X$, and $D_{X}$ be the sheaf of differential operators on $X$. We will need the following well known result.

Proposition 2.1. One has a natural isomorphism $\operatorname{Ext}_{D_{X}}^{i}\left(\mathcal{O}_{X}, \mathcal{O}_{X}\right) \cong H^{i}(X, \mathbb{C})$.

Proof. Let $D_{X}^{\bullet}$ be the dual D-module De Rham complex of $X$ :

$$
D_{X} \otimes_{\mathcal{O}_{X}} \Omega_{X}^{-d} \rightarrow \ldots \rightarrow D_{X} \otimes_{\mathcal{O}_{X}} \Omega_{X}^{-1} \rightarrow D_{X},
$$

where $\Omega_{X}^{-i}$ is the sheaf of polyvector fields of rank $i$ on $X$. Then $D_{X}^{\bullet}$ is a locally projective resolution of the D-module $\mathcal{O}_{X}$. Thus, the required Ext groups are equal to the hypercohomology groups of the complex of sheaves $\operatorname{Hom}_{D_{X}}\left(D_{X}^{\bullet}, \mathcal{O}_{X}\right)$. This complex is just the algebraic De Rham complex of $X$. But by Grothendieck's algebraic De Rham theorem, the hypercohomology of the De Rham complex is naturally isomorphic to the cohomology of $X$. This proves the proposition. 
Remark 2.2. Proposition 2.1 also holds for analytic varieties, with the same proof (using the usual De Rham theorem for complex manifolds).

Now let $X$ be a smooth algebraic variety over $\mathbb{C}$, and $g$ be an automorphism of $X$ of finite order. In this case the set of fixed points $X^{g}$ is a smooth algebraic variety, consisting of finitely many (say, $N_{g}$ ) connected components $X_{j}^{g}$ (possibly of different dimensions).

Assume that $X$ is affine, and let $D(X)$ be the algebra of differential operators on $X$. Then $g$ acts naturally as an automorphism of $D(X)$; namely, this action is induced by the action of $g$ on $\mathcal{O}(X)$ given by $(g f)(x):=f\left(g^{-1} x\right)$. Thus we can define the bimodule $D(X) g$ over $D(X)$ in an obvious way: $a \circ b g \circ c:=a b g(c) g$ for $a, b, c \in D(X)$.

The following proposition computes the Hochschild cohomology of $D(X)$ with coefficients in $D(X) g$.

Proposition 2.3. We have a natural isomorphism

$$
H H^{m}(D(X), D(X) g) \cong \oplus_{j=1}^{N_{g}} H^{m-2 \operatorname{codim} X_{j}^{g}}\left(X_{j}^{g}, \mathbb{C}\right) .
$$

In particular, this isomorphism is equivariant with respect to any automorphism of $X$ that commutes with $g$.

Proof. Let $\widetilde{D}_{X}$ and $\widetilde{D}_{X} g$ be the left D-modules on $X \times X$ corresponding to $D(X)$ and $D(X) g$, respectively, under the standard equivalence between left and right D-modules on the second factor $X$, see e.g. [Bo, HTT. Let $\Delta: X \rightarrow X \times X$ be the diagonal map, and $i: X \rightarrow X \times X$ be given by the formula $x \rightarrow\left(x, g^{-1} x\right)$. Then $\widetilde{D}_{X}=\Delta_{*} \mathcal{O}_{X}$ and $\widetilde{D}_{X} g=i_{*} \mathcal{O}_{X}$. Thus, using that $\Delta_{*}$ is left adjoint of $\Delta^{!}$, we have

$$
\begin{gathered}
H H^{m}(D(X), D(X) g)=\operatorname{Ext}_{D(X) \otimes D(X)^{\text {op }}}^{m}(D(X), D(X) g)=\operatorname{Ext}_{D_{X \times X}}^{m}\left(\widetilde{D}_{X}, \widetilde{D}_{X} g\right)= \\
=\operatorname{Ext}_{D_{X \times X}}^{m}\left(\Delta_{*} \mathcal{O}_{X}, i_{*} \mathcal{O}_{X}\right)=\operatorname{Ext}_{D_{X}}^{m}\left(\mathcal{O}_{X}, \Delta^{!} i_{*} \mathcal{O}_{X}\right) .
\end{gathered}
$$

Let $\eta_{j}: X_{j}^{g} \rightarrow X$ be the tautological embedding. Applying base change to the composition $\Delta ! i_{*}$ and using that $\eta_{j}^{!} \mathcal{O}_{X}=O_{X_{j}^{g}}\left[-\operatorname{codim} X_{j}^{g}\right]$, we have

$$
\operatorname{Ext}_{D_{X}}^{m}\left(\mathcal{O}_{X}, \Delta^{!} i_{*} \mathcal{O}_{X}\right)=\operatorname{Ext}_{D_{X}}^{m}\left(\mathcal{O}_{X}, \oplus_{j=1}^{N_{g}} \eta_{j *} \mathcal{O}_{X_{j}^{g}}\left[-\operatorname{codim} X_{j}^{g}\right]\right) .
$$

Since $\eta_{j}^{*}$ is left adjoint to $\eta_{j *}$, and $\eta_{j}^{*} \mathcal{O}_{X}=\mathcal{O}_{X_{j}^{g}}\left[+\operatorname{codim} X_{j}^{g}\right]$, we have

$$
\begin{aligned}
& \operatorname{Ext}_{D_{X}}^{m}\left(\mathcal{O}_{X}, \oplus_{j=1}^{N_{g}} \eta_{j *} \mathcal{O}_{X_{j}^{g}}\left[-\operatorname{codim} X_{j}^{g}\right]\right)= \\
& \oplus_{j=1}^{N_{g}} \operatorname{Ext}_{D_{X_{j}^{g}}}^{m}\left(\eta_{j}^{*} \mathcal{O}_{X}, \mathcal{O}_{X_{j}^{g}}\left[-\operatorname{codim} X_{j}^{g}\right]\right)= \\
& \oplus_{j=1}^{N_{g}} \operatorname{Ext}_{D_{X_{j}^{g}}}^{m}\left(\mathcal{O}_{X_{j}^{g}}\left[+\operatorname{codim} X_{j}^{g}\right], \mathcal{O}_{X_{j}^{g}}\left[-\operatorname{codim} X_{j}^{g}\right]\right)= \\
& =\oplus_{j=1}^{N_{g}} \operatorname{Ext}_{D_{X_{j}^{g}}}^{m-2 \operatorname{codim} X_{j}^{g}}\left(\mathcal{O}_{X_{j}^{g}}, \mathcal{O}_{X_{j}^{g}}\right)=\oplus_{j=1}^{N_{g}} H^{m-2 \operatorname{codim} X_{j}^{g}}\left(X_{j}^{g}, \mathbb{C}\right),
\end{aligned}
$$

where the last equality follows from Proposition 2.1. This implies the required statement.

For $X=\mathbb{A}^{n}$, Proposition 2.3 appears in [AFLS. We also note that an analog of Proposition 2.3 for $g=1$ and smooth real manifolds is due to Kassel and Mitschi (see e.g. BG]). 
Corollary 2.4. We have a natural isomorphism

$$
H H^{m}(G \ltimes D(X), G \ltimes D(X)) \cong\left(\oplus_{g \in G} \oplus_{j=1}^{N_{g}} H^{m-2 \operatorname{codim} X_{j}^{g}}\left(X_{j}^{g}, \mathbb{C}\right)\right)^{G} .
$$

Proof. It is well known (see e.g. AFLS) that for any algebra $A$ over a field of characteristic zero with an action of a finite group $G$, one has

$$
H H^{m}(G \ltimes A, G \ltimes A)=\left(\oplus_{g \in G} H H^{m}(A, A g)\right)^{G} .
$$

Indeed,

$$
H H^{m}(G \ltimes A, G \ltimes A)=\operatorname{Ext}_{(G \times G) \ltimes A \otimes A^{o p}}^{m}(G \ltimes A, G \ltimes A)
$$

By Shapiro's lemma, this equals

$$
\operatorname{Ext}_{G_{\text {diagonal }} \ltimes A \otimes A^{o p}}^{m}(A, G \ltimes A),
$$

which (by Maschke's theorem for $G$ ) is equal to

$$
\operatorname{Ext}_{A \otimes A^{\circ p}}^{m}(A, G \ltimes A)^{G}=H H^{m}(A, G \ltimes A)^{G},
$$

as desired.

So for $A=D(X)$, we find

$$
H H^{m}(G \ltimes D(X), G \ltimes D(X))=\left(\oplus_{g \in G} H H^{m}(D(X), D(X) g)\right)^{G} .
$$

The rest follows from Proposition 2.3 .

2.2. Twisted differential operators. Let us recall the theory of twisted differential operators (see [BB], section 2).

Let $X$ be a smooth affine algebraic variety over $\mathbb{C}$. Let $D(X)$ be the algebra of algebraic differential operators on $X$. Given a closed 2-form $\omega$ on $X$, we can define a two-cocycle on the Lie algebra $\operatorname{Vect}(X)$ with coefficients in the module of regular functions $\mathcal{O}(X)$, given by $v, w \rightarrow \omega(v, w)$. This cocycle defines an abelian extension of $\operatorname{Vect}(X)$ by $\mathcal{O}(X)$, which has an obvious structure of a Lie algebroid $\mathbb{L}_{\omega}$ over $X$. It is clear that this Lie algebroid depends only on the cohomology class of $\omega$, up to an isomorphism.

Let $U=U\left(\mathbb{L}_{\omega}\right)$ be the universal enveloping algebra of this Lie algebroid. For any $f \in \mathcal{O}(X)$, let $\widehat{f}$ be the corresponding section of $\mathbb{L}_{\omega}$. Let $I$ be the ideal in $U$ generated by the elements $\widehat{f}-f$. The quotient $U / I$ is the algebra of twisted differential operators $D_{\omega}(X)$.

More explicitly, $D_{\omega}(X)$ can be defined as the algebra generated by $\mathcal{O}(X)$ and "Lie derivatives" $\mathbf{L}_{v}, v \in \operatorname{Vect}(X)$, with defining relations

$$
f \mathbf{L}_{v}=\mathbf{L}_{f v},\left[\mathbf{L}_{v}, f\right]=L_{v} f,\left[\mathbf{L}_{v}, \mathbf{L}_{w}\right]=\mathbf{L}_{[v, w]}+\omega(v, w) .
$$

This algebra depends only on the cohomology class $[\omega]$ of $\omega$ (up to an isomorphism), and equals $D(X)$ if $\omega=0$.

An important special case of twisted differential operators is the algebra of differential operators on a line bundle. Namely, let $L$ be a line bundle on $X$. Since $X$ is affine, $L$ admits an algebraic connection $\nabla$ with curvature $\omega$, which is a closed 2 -form on $X$. Then it is easy to show that the algebra $D(X, L)$ of differential operators on $L$ is isomorphic to $D_{\omega}(X)$.

The classical analogs of twisted differential operator algebras are twisted cotangent bundles. In the case of an affine variety $X$, a twisted cotangent bundle is the usual cotangent bundle variety $T^{*} X$ equipped with the new symplectic structure $\Omega^{\prime}=\Omega+\pi^{*} \omega$, where $\Omega$ is the usual symplectic structure on $T^{*} X$ and $\pi: T^{*} X \rightarrow X$ 
is the projection. Here $\omega$ is a closed 2 -form on $X$. We will denote the twisted cotangent bundle by $T_{\omega}^{*} X$.

If the variety $X$ is smooth but not necessarily affine, then sheaves of algebras of twisted differential operators $D_{\psi, X}$ and twisted cotangent bundles $T_{\psi}^{*} X$ are classified by elements $\psi$ of the hypercohomology $H^{2}\left(X, \Omega_{X}^{\geq 1}\right)$, where $\Omega_{X}^{\geq 1}$ is the two-step complex of sheaves $\Omega_{X}^{1} \rightarrow \Omega_{X}^{2, c l}$, given by the De Rham differential acting from 1forms to closed 2-forms (sitting in degrees 1 and 2, respectively). If $X$ is projective then this space is isomorphic to $H^{2,0}(X, \mathbb{C}) \oplus H^{1,1}(X, \mathbb{C})$. We note that if $X$ is not affine then the twisted cotangent bundle is in general not a vector bundle but an affine space bundle, which need not be isomorphic to $T^{*} X$. We refer the reader to BB, Section 2, for details.

2.3. $D_{\omega}(X)$ as a universal deformation. Let $E$ be a subspace of the space of closed 2-forms on $X$ which projects isomorphically to $\left.H^{2}(X)\right]$ Then $D_{\omega}(X)$, $\omega \in E$, is a family of algebras parametrized by $H^{2}(X)$.

Lemma 2.5. Let $\omega \in E$. If the first order deformation $D_{\hbar \omega}(X)$ of $D(X)$ over $\mathbb{C}[\hbar] / \hbar^{2}$ is trivial, then $\omega=0$.

Proof. If the deformation is trivial then the module $\mathcal{O}(X)$ over $D(X)$ can be lifted to this deformation. This lifting must be trivial as a deformation of $\mathcal{O}(X)$-modules, i.e. it is isomorphic to $\mathcal{O}(X)[\hbar] / \hbar^{2}$ with the usual action of functions, and the action of vector fields by

$$
\mathbf{L}_{v} f=L_{v} f+\hbar A_{v}(f)
$$

for some operator $A_{v}$, where $L_{v} f$ is the usual Lie derivative. Since $\left[\mathbf{L}_{v}, f\right]=L_{v} f$ in $D_{\hbar \omega}(X)$, we find that $A_{v}(f)$ commutes with operators of multiplication by functions, so using that $\mathbf{L}_{g v}=g \mathbf{L}_{v}$, we get that $A_{v}(f)=\eta(v) f$, where $\eta$ is a 1-form on $X$. From this, using the commutation relation between $\mathbf{L}_{v}$ and $\mathbf{L}_{w}$, by a simple calculation we obtain $d \eta=\omega$. Since the map $E \rightarrow H^{2}(X)$ is an isomorphism, $\omega=0$. The lemma is proved.

By Proposition 2.3. $H H^{2}(D(X), D(X))=H^{2}(X)$. Thus using Lemma 2.5. we see that the 1-parameter formal deformations of $D(X)$ induced by $D_{\omega}(X), \omega \in E$, represent all elements of $H^{2}(X)$. Therefore, we get the following (apparently, well known) theorem.

Theorem 2.6. $D_{\omega}(X)$, where $\omega$ lies in the formal neighborhood of the origin in $E$, is a universal formal deformation of $D(X)$.

Remark 2.7. In fact, it is easy to show that the natural map $E \rightarrow H H^{2}(D(X), D(X)) \cong$ $H^{2}(X)$ induced by the deformation $D_{\omega}(X)$ is given by the formula $\omega \mapsto[\omega]$.

2.4. Algebro-geometric preliminaries. Let $Z$ be a smooth hypersurface in a smooth variety $X$. Let $i: Z \hookrightarrow X$ be the corresponding closed embedding. Let $N$ denote the normal bundle of $Z$ in $X$ (a line bundle). Let $\mathcal{O}_{X}(Z)$ denote the sheaf of regular functions on $X \backslash Z$ which have a pole of at most first order at $Z$. Then we have a natural map of $\mathcal{O}_{X}$-modules $\phi: \mathcal{O}_{X}(Z) \rightarrow i_{*} N$. Indeed, we have a natural residue map $\eta: \mathcal{O}_{X}(Z) \otimes_{\mathcal{O}_{X}} \Omega_{X}^{1} \rightarrow i_{*} \mathcal{O}_{Z}$ (where $\Omega_{X}^{1}$ is the sheaf of 1forms on $X$, and $i_{*}$ denotes the direct image of quasicoherent sheaves), hence a map

\footnotetext{
${ }^{1}$ Unless otherwise specified, cohomology of varieties is with complex coefficients.
} 
$\eta^{\prime}: \mathcal{O}_{X}(Z) \rightarrow i_{*} \mathcal{O}_{Z} \otimes_{\mathcal{O}_{X}} T X=i_{*}\left(\left.T X\right|_{Z}\right)$ (where $T X=\Omega_{X}^{-1}$ is the tangent bundle). The map $\phi$ is obtained by composing $\eta^{\prime}$ with the natural projection $\left.T X\right|_{Z} \rightarrow N$.

We have an exact sequence of $\mathcal{O}_{X}$-modules:

$$
0 \rightarrow \mathcal{O}_{X} \rightarrow \mathcal{O}_{X}(Z) \rightarrow i_{*} N \rightarrow 0
$$

(the third map is $\phi$ ). Thus we have a natural surjective map of $\mathcal{O}_{X}$-modules $\xi_{Z}: T X \rightarrow \mathcal{O}_{X}(Z) / \mathcal{O}_{X}$.

2.5. Cartan's lemma. We will need the following well known lemma, due to H. Cartan.

Lemma 2.8. (i) Let $X$ be a complex manifold with a holomorphic action of a finite group $G$, and $x \in X$ be a fixed point of $G$. Then there exists $G$-invariant open subsets $x \in U \subset X, 0 \in U_{0} \subset T_{x} X$ and a $G$-equivariant isomorphism $f: U_{0} \cong U$. In other words, locally near $x$ the $G$-action can be linearized.

(ii) (formal Cartan's lemma) Any action of a finite group $G$ on a formal polydisk over a field of characteristic zero is equivalent to a linear action.

Proof. For (i) see [C], p.97. For (ii), see e.g. [EM1, Lemma 7.8.

2.6. Rational Cherednik algebras. Recall the definition of rational Cherednik algebras (see e.g. [EG]).

Let $\mathfrak{h}$ be a finite dimensional complex vector space, and $G$ is a finite subgroup of $G L(\mathfrak{h})$. Let $S$ be the set of complex reflections in $G$, i.e., elements which have only one eigenvalue not equal to 1 . Let $t \in \mathbb{C}$, and $c: S \rightarrow \mathbb{C}$ be a $G$-invariant function. To this data one attaches an algebra $H_{t, c}(\mathfrak{h}, G)$, called the rational Cherednik algebra of $\mathfrak{h}, G$. It is generated by $G, \mathfrak{h}, \mathfrak{h}^{*}$ with defining relations

$$
g x g^{-1}={ }^{g} x, g y g^{-1}={ }^{g} y,\left[x, x^{\prime}\right]=\left[y, y^{\prime}\right]=0,
$$

and the main commutation relation

$$
[y, x]=t(y, x)-\sum_{s \in S} c_{s}\left(y, \alpha_{s}\right)\left(x, \alpha_{s}^{\vee}\right) s,
$$

where $x, x^{\prime} \in \mathfrak{h}^{*}, y, y^{\prime} \in \mathfrak{h}, \alpha_{s}$ is a nonzero linear function on $\mathfrak{h}$ vanishing on the fixed hyperplane of $s$ in $\mathfrak{h}$, and $\alpha_{s}^{\vee}$ is the element of $\mathfrak{h}$ vanishing on the fixed hyperplane of $s$ in $\mathfrak{h}^{*}$, such that $\left(\alpha_{s}, \alpha_{s}^{\vee}\right)=2$.

The algebra $H_{t, c}(\mathfrak{h}, G)$ has a natural representation in $M=\mathbb{C}[\mathfrak{h}]$ by DunklOpdam operators [DO]:

$$
x \mapsto x, g \mapsto g, y \mapsto D_{y}:=t \frac{\partial}{\partial y}+\sum_{s \in S} \frac{2 c_{s}}{1-\lambda_{s}} \frac{\left(\alpha_{s}, y\right)}{\alpha_{s}}(s-1)
$$

$\left(g \in G, x \in \mathfrak{h}^{*}, y \in \mathfrak{h}\right)$, where $\lambda_{s}$ is the nontrivial eigenvalue of $s$ in $\mathfrak{h}^{*}$.

This representation is faithful. Thus we can alternatively define $H_{t, c}(\mathfrak{h}, G)$ (for $t, c$ being variables) as follows: $H_{t, c}(\mathfrak{h}, G)$ is the subalgebra of the algebra $G \ltimes D(\mathfrak{h})_{r}[t, c]$ generated over $\mathbb{C}[t, c]$ by $\mathbb{C}[\mathfrak{h}]=S \mathfrak{h}^{*}, G$, and the operators $D_{y}$ (here $D(\mathfrak{h})_{r}$ is the algebra of differential operators on $\mathfrak{h}$ with rational coefficients).

The algebra $H_{t, c}(\mathfrak{h}, G)$ has an increasing filtration $F^{\bullet}$, defined by the rule $\operatorname{deg}(G)=$ $\operatorname{deg}\left(\mathfrak{h}^{*}\right)=0, \operatorname{deg}(\mathfrak{h})=1$. The PBW theorem (see e.g. EG] or [EM1, Subsection 3.2) says that $\operatorname{gr}_{F}\left(H_{t, c}(\mathfrak{h}, G)\right)=G \ltimes \mathbb{C}\left[\mathfrak{h} \oplus \mathfrak{h}^{*}\right]=G \ltimes S\left(\mathfrak{h}^{*} \oplus \mathfrak{h}\right)$. 
Let $\left\{y_{i}\right\}$ be a basis of $\mathfrak{h}$ and $\left\{x_{i}\right\}$ be the dual basis of $\mathfrak{h}^{*}$. Define the Euler element

$$
\mathbf{h}=\sum_{i} x_{i} y_{i}+\frac{\ell t}{2}-\sum_{s \in S} \frac{2 c_{s}}{1-\lambda_{s}} s \in H_{t, c}(\mathfrak{h}, G),
$$

where $\ell=\operatorname{dim} \mathfrak{h}$ (see GGOR]). Recall that $[\mathbf{h}, x]=x$ and $[\mathbf{h}, y]=-y$ for $x \in \mathfrak{h}^{*}$, $y \in \mathfrak{h}$.

Recall GGOR that the category $\mathcal{O}_{c}(\mathfrak{h}, G)$ for $H_{1, c}(\mathfrak{h}, G)$ is the category of representations of $H_{1, c}(\mathfrak{h}, G)$ which are direct sums of finite dimensional generalized eigenspaces of $\mathbf{h}$, with real part of the spectrum of $\mathbf{h}$ bounded from below. Thus, any module from $\mathcal{O}_{c}(\mathfrak{h}, G)$ is graded by generalized eigenvalues of $\mathbf{h}$. Note also that any $M \in \mathcal{O}_{c}(\mathfrak{h}, G)$ is finitely generated over the subalgebra $\mathbb{C}[\mathfrak{h}]$, and that $\mathcal{O}_{c}(\mathfrak{h}, G)$ may be alternatively defined as the category of $H_{1, c}(\mathfrak{h}, G)$-modules which are finitely generated over $\mathbb{C}[\mathfrak{h}]=S \mathfrak{h}^{*}$ and have a locally nilpotent action of $\mathfrak{h}$.

2.7. The formal completion of the rational Cherednik algebra. Now consider the degree-wise formal completion $\widehat{H}_{t, c}(\mathfrak{h}, G)$ of the rational Cherednik algebra $H_{t, c}(\mathfrak{h}, G)$, i.e., its restriction to the formal neighborhood of zero in $\mathfrak{h}$ as a $\mathbb{C}[\mathfrak{h}]$-module: $\widehat{H}_{t, c}(\mathfrak{h}, G):=\mathbb{C}[[\mathfrak{h}]] \otimes_{\mathbb{C}[\mathfrak{h}]} H_{t, c}(\mathfrak{h}, G)$. This completion has a natural algebra structure, and comes with an increasing filtration $F^{\bullet}$ defined by the rule $F^{i} \widehat{H}_{t, c}(\mathfrak{h}, G)=\mathbb{C}[[\mathfrak{h}]] \otimes_{\mathbb{C}[\mathfrak{h}]} F^{i} H_{t, c}(\mathfrak{h}, G)$.

If $M \in \mathcal{O}_{c}(\mathfrak{h}, G)$, then the completion $\widehat{M}$ of $M$ by the grading defined by eigenvalues of $\mathbf{h}$ is a representation of the algebra $\widehat{H}_{t, c}(\mathfrak{h}, G)$. The category $\widehat{\mathcal{O}}_{c}(\mathfrak{h}, G)$ is defined as the category of modules over the algebra $\widehat{H}_{t, c}(\mathfrak{h}, G)$ of the form $\widehat{M}$. Then the functor $\mathcal{O}_{c}(\mathfrak{h}, G) \rightarrow \widehat{\mathcal{O}}_{c}(\mathfrak{h}, G)$ given by $M \mapsto \widehat{M}$ is an equivalence of categories (see $\mathrm{BE}$, Theorem 2.3).

We will need the following result.

Proposition 2.9. (see also $\overline{\mathrm{BE}}$, Theorem 2.3) The category $\widehat{\mathcal{O}}_{c}(\mathfrak{h}, G)$ coincides with the category of $\widehat{H}_{1, c}(\mathfrak{h}, G)$-modules which are finitely generated over $\mathbb{C}[[\mathfrak{h}]]$.

Proof. It is clear that the first category is a full subcategory of the second one; so our job is to show that any $\widehat{H}_{1, c}(\mathfrak{h}, G)$-module $N$ which is finitely generated over $\mathbb{C}[[\mathfrak{h}]]$ belongs to $\mathcal{O}_{c}(\mathfrak{h}, G)$.

Let $I$ be the maximal ideal in $\mathbb{C}[[\mathfrak{h}]]$. The module $N$ has a decreasing filtration $N \supset I N \supset I^{2} N \supset \ldots$, , such that the quotients $N / I^{k} N$ are finite dimensional, and $N$ is the inverse limit of $N / I^{k} N$. The element $\mathbf{h}$ normalizes $I$, and adh has positive integer eigenvalues on $I$. This implies that for any $\lambda \in \mathbb{C}$ the generalized eigenspaces $\left(N / I^{k} N\right)(\lambda)$ form a projective system which stabilizes at some $k=k(\lambda)$. In particular, $N(\lambda)$ is a finite dimensional space which coincides with $\left(N / I^{k} N\right)(\lambda)$ for large $k$. Thus, the spectrum of $\mathbf{h}$ in $N$ is bounded from below (by the minimum of real parts of its eigenvalues on $N / I N)$, and $N=\widehat{M}$, where $M$ is the direct sum of $N(\lambda)$. It is clear that $M \in \mathcal{O}_{c}(\mathfrak{h}, G)$. The proposition is proved.

Remark 2.10. Note that Proposition2.9 is false for uncompleted algebras $H_{1, c}(\mathfrak{h}, G)$. Proposition 2.9 is analogous to the statement that any $D$-module on a formal polydisk $X$ which is finitely generated as a module over $\mathcal{O}(X)$ is a multiple of $\mathcal{O}(X)$, which also fails in the uncompleted case, e.g. for $X=\mathbb{A}^{1}$.

One can also define the completion of $\widehat{H}_{t, c}(\mathfrak{h}, G)_{z}$ at any point $z \in \mathfrak{h} / W$, so that $\widehat{H}_{t, c}(\mathfrak{h}, G)=\widehat{H}_{t, c}(\mathfrak{h}, G)_{0}$. This gives a certain centralizer algebra, as discussed 
in $\mathrm{BE}$, Subsection 3.3. See also Subsection 2.9 below for a discussion of this construction in the more general nonlinear case.

2.8. The Cherednik algebra of a variety with a finite group action. We will now generalize the definition of $H_{t, c}(\mathfrak{h}, G)$ to the case of a nonlinear action of $G$. Let $X$ be an affine algebraic variety over $\mathbb{C}$, and $G$ be a finite group of automorphisms of $X$. Let $X^{g} \subset X$ be the set of fixed points of $g \in G$. A component $Y$ of $X^{g}$ of codimension 1 in $X$ will be called a reflection hypersurface. For instance, if $X=\mathfrak{h}$ is a vector space and $G$ acts linearly, then $X^{g} \subset X$ is a subspace, and if $\operatorname{codim} X^{g}=1$ then $g$ is a complex reflection and $X^{g}$ is the corresponding reflection hyperplane, which justifies the terminology.

Let $E$ be a $G$-invariant subspace of the space of closed 2-forms on $X$, which projects isomorphically to $H^{2}(X)$ (it is clear that $E$ exists). Consider the algebra $G \ltimes \mathcal{O}\left(T^{*} X\right)$, where $T^{*} X$ is the cotangent bundle of $X$. We are going to define a deformation $H_{t, c, \omega}(X, G)$ of this algebra parametrized by

1) complex numbers $t$,

2) $G$-invariant functions $c$ on the (finite) set $S$ of pairs $s=(Y, g)$, where $g \in G$, $g \neq 1$, and $Y \subset X^{g}$ is a reflection hypersurface, and

3) elements $\omega \in E^{G}=H^{2}(X)^{G}$.

If all the parameters are zero, this algebra will coincide with $G \ltimes \mathcal{O}\left(T^{*} X\right)$.

Let $t, c=\{c(Y, g)\}, \omega \in E^{G}$ be variables. Let $D_{\omega / t}(X)_{r}$ be the algebra (over $\mathbb{C}\left[t, t^{-1}, \omega\right]$ ) of twisted (by $\left.\omega / t\right)$ differential operators on $X$ with rational coefficients.

Definition 2.11. A Dunkl-Opdam operator for $X, G$ is an element of $G \ltimes D_{\omega / t}(X)_{r}[c]$ given by the formula

$$
D:=t \mathbf{L}_{v}+\sum_{(Y, g) \in S} \frac{2 c(Y, g)}{1-\lambda_{Y, g}} \cdot f_{Y}(x) \cdot(g-1),
$$

where $\lambda_{Y, g}$ is the eigenvalue of $g$ on the conormal bundle to $Y, v \in \Gamma(X, T X)$ is a vector field on $X$, and $f_{Y} \in \Gamma\left(X, \mathcal{O}_{X}(Z)\right)$ is an element of the coset $\xi_{Y}(v) \in$ $\Gamma\left(X, \mathcal{O}_{X}(Z) / \mathcal{O}_{X}\right)$ (recall that $\xi_{Y}$ is defined in Subsection 2.4).

Definition 2.12. The algebra $H_{t, c, \omega}(X, G)$ is the subalgebra of $D_{\omega / t}(X)_{r}[c]$ generated (over $\mathbb{C}[t, c, \omega]$ ) by the function algebra $\mathcal{O}_{X}$, the group $G$, and the DunklOpdam operators.

By specializing $t, c, \omega$ to numerical values, we can define a family of algebras over $\mathbb{C}$, which we will also denote $H_{t, c, \omega}(X, G)$. Note that when we set $t=0$, the term $t \mathbf{L}_{v}$ does not become 0 but turns into the classical momentum, as in EM1, Subsection 2.10.

Definition 2.13. $H_{t, c, \omega}(X, G)$ is called the Cherednik algebra of $X, G$.

Example 2.14. $X=\mathfrak{h}$ is a vector space and $G$ is a subgroup in $G L(\mathfrak{h})$. Let $v$ be a constant vector field, and let $f_{Y}(x)=\left(\alpha_{Y}, v\right) / \alpha_{Y}(x)$, where $\alpha_{Y} \in \mathfrak{h}^{*}$ is a nonzero functional vanishing on $Y$. Then the operator $D$ is just the usual DunklOpdam operator $D_{v}$ in the complex reflection case (see Subsection 2.6). This implies that all the Dunkl-Opdam operators in the sense of Definition 2.11 have the form $\sum f_{i} D_{y_{i}}+a$, where $f_{i} \in \mathbb{C}[\mathfrak{h}], a \in G \ltimes \mathbb{C}[\mathfrak{h}]$, and $D_{y_{i}}$ are the usual Dunkl-Opdam operators (for some basis $y_{i}$ of $\mathfrak{h}$ ). So the algebra $H_{t, c}(\mathfrak{h}, G)=H_{t, c, 0}(X, G)$ is the rational Cherednik algebra for $\mathfrak{h}, G$, see Subsection 2.6. 
The algebra $H_{t, c, \omega}(X, G)$ has a filtration $F^{\bullet}$ which is defined on generators by $\operatorname{deg}\left(\mathcal{O}_{X}\right)=\operatorname{deg}(G)=0, \operatorname{deg}(D)=1$ for Dunkl-Opdam operators $D$.

Proposition 2.15. Let $\delta \neq 0$ be a G-invariant regular function on $X$ which vanishes on reflection hypersurfaces, and let $X^{\circ}$ be the complement in $X$ of the zero set of $\delta$. Then we have natural filtration-compatible isomorphisms

$$
H_{t, c, \omega}(X, G)\left[\delta^{-1}\right] \cong H_{t, c, \omega}\left(X^{\circ}, G\right) \cong H_{t, 0, \omega}\left(X^{\circ}, G\right) \cong H_{t, 0, \omega}(X, G)\left[\delta^{-1}\right] .
$$

In particular, if $t \neq 0$ then $H_{t, c, \omega}(X, G)\left[\delta^{-1}\right] \cong G \ltimes D_{\omega / t}\left(X^{\circ}\right)$ and $H_{0, c, \omega}(X, G)\left[\delta^{-1}\right] \cong$ $G \ltimes \mathcal{O}\left(T_{\omega}^{*} X^{\circ}\right)$.

Proof. This follows immediately from the definition.

2.9. The formal completion of $H_{t, c, \omega}(X, G)$ at $z \in X / G$. Let us now define the formal completion of $H_{t, c, \omega}(X, G)$ at a point $z \in X / G$, generalizing the definition of Subsection 2.7 to the nonlinear case. We will assume that the parameters $t, c, \omega$ are numerical; the case when they are variables is similar.

By the Hilbert-Noether theorem, $X / G=\operatorname{Spec} \mathcal{O}(X)^{G}$ is an affine variety (i.e., the algebra $\mathcal{O}(X)^{G}$ is finitely generated), and $F^{i} H_{t, c, \omega}(X, G)$ is a finitely generated $\mathcal{O}(X)^{G}$-module for each $i$ (under left or right multiplication).

Let $z \in X / G$. Then we can consider the degree-wise completion

$$
\widehat{H}_{t, c, \omega}(X, G)_{z}:=\widehat{\mathcal{O}(X / G)_{z}} \otimes_{\mathcal{O}(X / G)} H_{t, c, \omega}(X, G),
$$

where $\widehat{\mathcal{O}(X / G)}$ is the algebra of regular functions on the formal neighborhood of $z$ in $X / G$. This is naturally an algebra, with a filtration $F^{\bullet}$ such that

$$
F^{i} \widehat{H}_{t, c, \omega}(X, G)_{z}=\widehat{\mathcal{O}(X / G)_{z}} \otimes_{\mathcal{O}(X / G)} F^{i} H_{t, c, \omega}(X, G) .
$$

Moreover, it turns out that this algebra can be described explicitly via ordinary rational Cherednik algebras.

Namely, let $x \in X$ be a preimage of $z$, let $G_{x} \subset G$ be the stabilizer of $x$, and let $U_{x}$ be the formal neighborhood of $x$ in $X$. Then $G_{x}$ acts on $U_{x}$. By Lemma 2.8 any action of a finite group on a formal polydisk over $\mathbb{C}$ is equivalent to a linear action, thus the action of $G_{x}$ on $U_{x}$ is equivalent to the linear representation of $G_{x}$ on $\mathfrak{h}:=T_{x} U_{x}$. Let $P=\operatorname{Fun}_{G_{x}}\left(G, \widehat{H}_{t, c}\left(\mathfrak{h}, G_{x}\right)\right)$ be the space of functions invariant under left multiplication by elements of $G_{x}$. Then $P$ is a free right $\widehat{H}_{t, c}\left(\mathfrak{h}, G_{x}\right)$ module of rank $N:=\left|G / G_{x}\right|$. Let $Z\left(G, G_{x}, \widehat{H}_{t, c}\left(\mathfrak{h}, G_{x}\right)\right):=\operatorname{End}_{\widehat{H}_{t, c}\left(\mathfrak{h}, G_{x}\right)}(P)$ be the corresponding centralizer algebra, see $[\mathrm{BE}$, Subsection 3.2; it is non-canonically isomorphic to $\operatorname{Mat}_{N}\left(\widehat{H}_{t, c}\left(\mathfrak{h}, G_{x}\right)\right)$.

Now note that the terms in the Dunkl-Opdam operators (1) corresponding to elements $g \in G \backslash G_{x}$ are regular at $x$. Therefore, analogously to $[\mathrm{BE}$, Theorem 3.2, we have

Proposition 2.16. There is a natural filtered isomorphism

$$
\widehat{H}_{t, c, \omega}(X, G)_{z} \cong Z\left(G, G_{x}, \widehat{H}_{t, c}\left(\mathfrak{h}, G_{x}\right)\right) .
$$

2.10. The PBW theorem for $H_{t, c, \omega}(X, G)$. We will now prove the PBW theorem for $H_{t, c, \omega}(X, G)$. We will assume $t, c, \omega$ are variables.

Theorem 2.17. (the $P B W$ theorem) We have a natural isomorphism

$$
\operatorname{gr}_{F}\left(H_{t, c, \omega}(X, G)\right) \cong G \ltimes \mathcal{O}\left(T^{*} X\right)[t, c, \omega] .
$$


This implies that for numerical $t, c, \omega$, we have a natural isomorphism

$$
\operatorname{gr}_{F}\left(H_{t, c, \omega}(X, G)\right) \cong G \ltimes \mathcal{O}\left(T^{*} X\right) .
$$

Proof. Suppose first that $X=\mathfrak{h}$ is a vector space and $G$ is a subgroup in $G L(\mathfrak{h})$. Then, as we mentioned, $H_{t, c, \omega}(X, G)$ is the rational Cherednik algebra for $G$. So in this case the theorem is true, see [EM1, Subsection 3.2.

Now consider arbitrary $X$. Let us define a homomorphism of graded algebras

$$
\psi: \operatorname{gr}_{F}\left(H_{t, c, \omega}(X, G)\right) \rightarrow G \ltimes \mathcal{O}\left(T^{*} X\right)[t, c, \omega]
$$

(the principal symbol homomorphism). To this end, consider another filtration $\Phi^{\bullet}$ on $H_{t, c, \omega}(X, G)$, obtained by restricting the usual filtration by order of differential operators from $G \ltimes D_{\omega / t}(X)_{r}[c]$ to $H_{t, c, \omega}(X, G)$. Then $F^{m} \subset \Phi^{m}$, since the DunklOpdam operators have degree 1 under $\Phi$. Thus, for any $a \in F^{m} / F^{m-1}$ we can define $\psi(a)$ to be the image of $a$ in $\Phi^{m} / \Phi^{m-1} \subset O\left(T^{*} X\right)_{r}[t, c, \omega]$ (where $O\left(T^{*} X\right)_{r}$ are rational functions on $T^{*} X$ polynomial on the fibers). More explicitly, take $a=g D_{v_{1}} \ldots D_{v_{m}}, g \in G, v_{i} \in \Gamma(X, T X)$ (any element of $F_{m}$ is a linear combination of elements of this form). Then $\psi(a)=g v_{1} \ldots v_{m}$. Hence, $\psi(a)$ in fact belongs to $G \ltimes \mathcal{O}\left(T^{*} X\right)[t, c, \omega]$ (i.e., has no poles), i.e., $\psi$ is well defined.

The homomorphism $\psi$ is surjective, since $G \ltimes O\left(T^{*} X\right)$ is spanned by elements of the form $g v_{1} \ldots v_{m}$. Thus, our job is to show that $\psi$ is injective (this is the nontrivial part of the proof).

Recall that for an affine variety $Y$ and a morphism $f: M \rightarrow N$ of finitely generated $\mathcal{O}(Y)$-modules, $f$ is injective iff it is injective on the formal neighborhood of each point of $Y$. Indeed, taking $K=\operatorname{Ker} f$ (a finitely generated $\mathcal{O}(Y)$-module by the Hilbert basis theorem), we find that the completion $K_{y}$ of $K$ at each $y \in Y$ is zero, which implies that the fiber $\left.K\right|_{y}$ of $K$ at each $y \in Y$ is zero, giving $K=0$ by Nakayama's lemma.

In each degree, $\psi$ is a morphism of finitely generated modules over $\mathcal{O}(X)^{G}[t, c, \omega]=$ $\mathcal{O}(X / G)[t, c, \omega]$. Therefore, to check the injectivity of $\psi$, it suffices to check the injectivity of $\psi$ on the formal neighborhood of each point $z \in X / G$.

Let $x$ be a preimage of $z$ in $X$, and $G_{x}$ be the stabilizer of $x$ in $G$. Then $G_{x}$ acts on the formal neighborhood $U_{x}$ of $x$ in $X$, and by Lemma 2.8, this action is equivalent to the linear action of $G_{x}$ on $\mathfrak{h}=T_{x} U_{x}$. Therefore, by Proposition 2.16, the restriction of the map $\psi$ to the formal neighborhood of $z$ may be identified with the map

$$
\widehat{\psi}: \operatorname{gr}_{F} Z\left(G, G_{x}, \widehat{H}_{t, c}\left(\mathfrak{h}, G_{x}\right)\right)[\omega] \rightarrow Z\left(G, G_{x}, G_{x} \ltimes \widehat{\mathcal{O}}\left(T^{*} \mathfrak{h}\right)\right)[t, c, \omega]
$$

induced by the map

$$
\bar{\psi}: \operatorname{gr}_{F} \widehat{H}_{t, c}\left(\mathfrak{h}, G_{x}\right)[\omega] \rightarrow G_{x} \ltimes \widehat{\mathcal{O}}\left(T^{*} \mathfrak{h}\right)[t, c, \omega],
$$

where $\widehat{\mathcal{O}}\left(T^{*} \mathfrak{h}\right):=\mathbb{C}[[\mathfrak{h}]] \otimes_{\mathbb{C}[\mathfrak{h}]} \mathcal{O}\left(T^{*} \mathfrak{h}\right)=\mathbb{C}[[\mathfrak{h}]]\left[\mathfrak{h}^{*}\right]$. Clearly, the injectivity of $\widehat{\psi}$ is equivalent to the injectivity of $\bar{\psi}$. But the map $\bar{\psi}$ is obtained by completing at the origin the map $\psi$ for $X$ replaced by $\mathfrak{h}$ and $G$ by $G_{x}$. Therefore, it suffices to prove the injectivity of $\psi$ in the linear case, which has been accomplished already. We are done.

Remark 2.18. The following remark is meant to clarify the proof of Theorem 2.17. In the case $X=\mathfrak{h}$, the proof of Theorem 2.17 is based, essentially, on the (fairly nontrivial) fact that the usual Dunkl-Opdam operators $D_{v}$ commute with 
each other. It is therefore very important to note that in contrast with the linear case, for a general $X$ we do not have any natural commuting family of DunklOpdam operators. Instead, the operators (11) satisfy a weaker property, which is still sufficient for the PBW theorem. This property says that if $D_{1}, D_{2}, D_{3}$ are Dunkl-Opdam operators corresponding to vector fields $v_{1}, v_{2}, v_{3}:=\left[v_{1}, v_{2}\right]$ and some choices of the functions $f_{Y}$, then $\left[D_{1}, D_{2}\right]-D_{3} \in G \ltimes \mathcal{O}(X)$ (i.e., it has no poles). To prove this property, it is sufficient to consider the case when $X$ is a formal polydisk, with a linear action of $G$. But in this case everything follows from the commutativity of the "classical" Dunkl-Opdam operators $D_{v}$.

Remark 2.19. Suppose $G=1$. Then for $t \neq 0, H_{t, \omega}(X, G)=D_{\omega / t}(X)$. On the other hand, $H_{0, \omega}(X, G)$ is the Poisson algebra $\mathcal{O}\left(T_{\omega}^{*} X\right)$ (in which the Poisson bracket is induced by the $t$-deformation).

Remark 2.20. For any $G, H_{0,0, \omega}(X, G)=G \ltimes \mathcal{O}\left(T_{\omega}^{*} X\right)$, and $H_{1,0, \omega}(X, G)=$ $G \ltimes D_{\omega}(X)$. Also, if $\lambda \neq 0$ then $H_{\lambda t, \lambda c, \lambda \omega}(X, G)=H_{t, c, \omega}(X, G)$.

Remark 2.21. The construction of $H_{t, c, \omega}(X, G)$ and the PBW theorem extend in a straightforward manner to the case when the ground field is not $\mathbb{C}$ but an algebraically closed field $k$ of positive characteristic, provided that the order of the group $G$ is relatively prime to the characteristic.

\subsection{Cherednik algebra as a universal deformation.}

Lemma 2.22. If for some $(c, \omega)$ the first order deformation $H_{1, \hbar c, \hbar \omega}(X, G)$ of $G \ltimes D(X)$ over $\mathbb{C}[\hbar] / \hbar^{2}$ is trivial then $c=0, \omega=0$.

Proof. Localizing the algebra to formal neighborhoods of points of $X / G$ using Proposition 2.16, and using that the lemma is true in the linear case (Theorem 2.16 in [EG]), we obtain that $c=0$. The rest follows from Lemma 2.5,

Theorem 2.23. The algebra $H_{1, c, \omega}(X, G)$ (with formal $c$ and $\omega$ ) is a universal formal deformation of $H_{1,0,0}(X, G)=G \ltimes D(X)$.

Proof. From Corollary 2.4, we get:

Proposition 2.24. One has

$$
H H^{2}(G \ltimes D(X), G \ltimes D(X))=H^{2}(X)^{G} \oplus\left(\oplus_{(Y, g) \in S} H^{0}(Y)\right)^{G} .
$$

(Note that $H^{0}(Y)=\mathbb{C}$; we wrote $H^{0}(Y)$ to make more obvious the action of $G$.) Thus, the dimension of $H H^{2}(G \ltimes D(X), G \ltimes D(X))$ is the same as the dimension of the space of parameters $(c, \omega)$. Therefore, Theorem 2.23 follows immediately from Lemma 2.22

Remark 2.25. In fact, it can be shown (by reducing to the linear case using formal completions) that the map

$E^{G} \oplus \operatorname{Fun}(X, \mathbb{C})^{G} \rightarrow H H^{2}(G \ltimes D(X), G \ltimes D(X))=\left(\oplus_{(Y, g) \in S} H^{0}(Y)\right)^{G} \oplus H^{2}(X)^{G}$ induced by the deformation $H_{1, c, \omega}(X, G)$ is given by $(c, \omega) \mapsto(c,[\omega])$.

Remark 2.26. A special case of the construction of $H_{t, c, \omega}(X, G)$ is as follows. Let $L$ be a $G$-equivariant line bundle on $X$. Define $H_{t, c}^{L}(X, G)$ to be the algebra generated by $\mathcal{O}(X), G$, and the Dunkl-Opdam operators regarded as elements of the smash product of $G$ with the algebra of differential operators on $L$ with rational coefficients. It is easy to see that $H_{t, c}^{L}(X, G)=H_{t, c, t \omega}(X, G)$, where $\omega$ is the curvature of a $G$-stable connection on $L$ (which always exists since $X$ is affine). 
Remark 2.27. Assume $G=1$. Consider the family of algebras $H_{t, \omega / t}(X):=$ $H_{t, 0, \omega / t}(X, G)$. As $t \rightarrow 0$, this algebra can be naturally degenerated into the algebra $H_{\infty}(X, \omega)$ defined by generators $f \in \mathcal{O}(X)$ and $p_{v}, v \in \operatorname{Vect}(X)$, with defining relations

$$
f p_{v}=p_{f v}=p_{v} f,\left[p_{v}, p_{w}\right]=\omega(v, w) .
$$

Thus, $H_{\infty}(X, \omega)$ is a quantization of the (degenerate) Poisson structure on $T^{*} X$, whose Poisson bracket is defined by the formula

$$
\{f, g\}=\pi^{*} \omega\left(v_{f}, v_{g}\right),
$$

where $f, g \in \mathcal{O}\left(T^{*} X\right)$, and $v_{f}, v_{g}$ the corresponding Hamiltonian vector fields. The algebra $\mathcal{O}\left(T^{*} X\right)$ with this Poisson structure is obtained as the limit of $H_{0, \omega / t}(X)$ when $t$ goes to zero.

Remark 2.28. The above results generalize without significant changes to the case when the group $G$ acts on $X$ in a not necessarily faithful manner. In this case, let $K$ be the kernel of this action, so that $G / K \subset \operatorname{Aut}(X)$. Then the algebra $H_{t, c, \omega}(X, G)$ is defined as above, except that

$$
\omega \in\left(H^{2}(X) \otimes \mathbb{C}[K]\right)^{G}=\left(H^{2}(X) \otimes \mathbb{C}[K]^{K}\right)^{G / K}
$$

is a $G / K$-invariant 2 -form on $X$ with values in the center $\mathbb{C}[K]^{K}$ of the group algebra of $K$. The algebra $H_{t, c, \omega}(X, G)$ satisfies the PBW theorem and $H_{1, c, \omega}(X, G)$ is a universal deformation of $H_{1,0,0}(X, G)=G \ltimes D(X)$. These results are proved similarly to the case of the faithful action.

2.12. The spherical subalgebra and the center of the Cherednik algebra. Let $\mathrm{e}=|G|^{-1} \sum_{g \in G} g$ be the symmetrizing idempotent of $G$. Then we can define the spherical subalgebra $H_{t, c, \omega}(X, G)$ e of $H_{t, c, \omega}(X, G)$. We denote by $Z_{0, c, \omega}(X, G)$ the center of $H_{0, c, \omega}(X, G)$.

Theorem 2.29. (i) $\operatorname{gr}_{F}\left(Z_{0, c, \omega}(X, G)\right)=Z_{0,0,0}(X, G)=\mathcal{O}\left(T^{*} X / G\right)$.

(ii) (Satake isomorphism) The map $z \rightarrow z$ e gives an isomorphism $Z_{0, c, \omega}(X, G) \rightarrow$ $\mathrm{e} H_{0, c, \omega}(X, G) \mathrm{e}$. In particular, $\mathrm{e} H_{0, c, \omega}(X, G) \mathrm{e}$ is a commutative algebra.

Proof. (i) First of all, the result holds for $c=0$, since $Z_{0,0, \omega}(X, G)=\mathcal{O}\left(T_{\omega}^{*} X / G\right)$. In general, the PBW theorem for $H_{t, c, \omega}(X, G)$ implies that we have a natural degreepreserving inclusion $\iota: \operatorname{gr}_{F}\left(Z_{0, c, \omega}(X, G)\right) \hookrightarrow Z_{0,0,0}(X, G)$. Assume that $\iota$ is not an isomorphism. Then the filtered deformation of $H_{0,0,0}(X, G)$ into $H_{0, c, \omega}(X, G)$ induces a nonzero Poisson bracket $\{$,$\} of some negative degree d$ on $Z_{0,0,0}(X, G)=$ $\mathcal{O}\left(T^{*} X / G\right)$. On the other hand, by Proposition 2.15 and the special case $c=0$, the corresponding inclusion $\iota^{\prime}: \operatorname{gr}_{F}\left(Z_{0, c, \omega}\left(X^{\circ}, G\right)\right) \hookrightarrow Z_{0,0,0}\left(X^{\circ}, G\right)$ is an isomorphism. Hence, the corresponding Poisson bracket $\{,\}^{\prime}$ of degree $d$ on $Z_{0,0,0}\left(X^{\circ}, G\right)=$ $\mathcal{O}\left(T^{*} X^{\circ} / G\right)$ vanishes. But the Poisson bracket $\{$,$\} is the restriction of \{,\}^{\prime}$ to a subalgebra, so it must vanish as well. This is a contradiction. Thus, $\iota$ is an isomorphism, proving (i).

Part (ii) follows from (i), since (i) means that the associated graded of the map (ii) is an isomorphism.

Let $M_{c, \omega}(X, G)$ be the spectrum of $Z_{0, c, \omega}(X, G)$. It follows from the above that $M_{c, \omega}(X, G)$ is an irreducible Poisson variety (the Poisson structure comes from the deformation of $H_{0, c, \omega}(X, G)$ e into $\left.\mathrm{e} H_{t, c, \omega}(X, G) \mathrm{e}\right)$. We also have a Lagrangian $\operatorname{map} \pi: M_{c, \omega}(X, G) \rightarrow X / G$, whose generic fiber is $T_{x}^{*} X$. 
Example 2.30. $M_{0, \omega}(X, G)=T_{\omega}^{*} X / G$.

Note that the variety structure of $M_{c, \omega}(X, G)$ is independent of $\omega$, and only the Poisson bracket depends of $\omega$. In fact, the dependence of the Poisson bracket on $M_{c, \omega}(X, G)$ on $\omega$ is given by the formula

$$
\{f, g\}_{\omega}=\{f, g\}_{0}+\pi^{*} \omega\left(v_{f}, v_{g}\right)
$$

where $v_{f}, v_{g}$ are the Hamiltonian vector fields corresponding to functions $f, g$.

2.13. Globalization. Now let $X$ be any smooth algebraic variety, and $G \subset \operatorname{Aut}(X)$. Assume that $X$ admits a cover by affine $G$-invariant open sets. Then the quotient variety $X / G$ exists.

For any affine open set $U$ in $X / G$, let $U^{\prime}$ be the preimage of $U$ in $X$. Then we can define the algebra $H_{t, c, 0}\left(U^{\prime}, G\right)$ as above. If $U \subset V$, we have an obvious restriction map $H_{t, c, 0}\left(V^{\prime}, G\right) \rightarrow H_{t, c, 0}\left(U^{\prime}, G\right)$. The gluing axiom is clearly satisfied. Thus the collection of algebras $H_{t, c, 0}\left(U^{\prime}, G\right)$ can be extended (by sheafification) to a quasicoherent sheaf of algebras on $X / G$. We are going to denote this sheaf by $H_{t, c, 0, X, G}$ and call it the sheaf of Cherednik algebras on $X / G$. Thus, $H_{t, c, 0, X, G}(U)=$ $H_{t, c, 0}\left(U^{\prime}, G\right)$.

Similarly, if $\psi \in H^{2}\left(X, \Omega_{\bar{X}}^{\geq 1}\right)^{G}$, we can define the sheaf of twisted Cherednik algebras $H_{t, c, \psi, X, G}$. This is done similarly to the case of twisted differential operators (which is the case $G=1$ ). In particular, if $L$ is a $G$-equivariant line bundle on $X$, then we can define the sheaf of algebras $H_{t, c, X, G}^{L}$ in the obvious way (it is glued out of the algebras $\left.H_{t, c}^{L}\left(U^{\prime}, G\right)\right)$.

Analogously, the varieties $M_{c, \psi}\left(U^{\prime}, G\right)$ can be glued into a single variety $M_{c, \psi}(X, G)$, which is Poisson and has a surjective Lagrangian projection $\pi: M_{c, \psi}(X) \rightarrow X / G$.

Definition 2.31. The variety $M_{c, \psi}(X, G)$ is called the Calogero-Moser space of $X$.

Example. $M_{0, \psi}(X, G)=T_{\psi}^{*} X / G$, the quotient of the twisted cotangent bundle of $X(\overline{\mathrm{BB}}$, Section 2$)$ by the $G$-action.

Remark 2.32. These constructions can be generalized to the case when $X$ does not necessarily admit a cover by $G$-invariant affine open sets. In this case $X / G$ is an algebraic space which may not be a scheme $(\overline{\mathrm{dJ}})$. But the above constructions go through if instead of $G$-equivariant affine open subsets $U^{\prime}$ in $X$ (=affine open subsets $U$ in $X / G$ ) we use $G$-equivariant étale morphisms $\pi: U^{\prime} \rightarrow X$, where $U^{\prime}$ is affine (such a morphism descends to an étale morphism $\bar{\pi}: U \rightarrow X / G$, where $U=U^{\prime} / G$ is affine). Thus, we obtain a sheaf of algebras $H_{t, c, \psi, X, G}$ on the algebraic space $X / G$ in the étale topology.

2.14. Modified Cherednik algebras. Let $X$ is a smooth algebraic variety, and $\psi \in H^{2}\left(X, \Omega_{\bar{X}}^{\geq 1}\right)$ be a twisting for differential operators on $X$. Let $S$ be a finite set of smooth divisors $Y \subset X$, and let $X^{\circ}$ be the complement of these divisors. Let $\eta: S \rightarrow \mathbb{C}$ be a function. Denote by $D_{\eta, \psi, X}$ the sheaf of algebras locally generated inside $D_{\psi, X}$ 。 by regular functions and elements $D=\mathbf{L}_{v}+\sum_{Y \in S} \eta(Y) f_{Y}(x)$, where $v \in \Gamma(X, T X)$ and $f_{Y}$ is a section of $\mathcal{O}_{X}(Y) / \mathcal{O}_{X}$ representing $v$. Also, let $\psi_{Y}$ be the class in $H^{2}\left(X, \Omega_{X}^{\geq 1}\right)$ defined by the line bundle $\mathcal{O}_{X}(Y)^{-1}$, whose sections are functions vanishing on $Y$. 
Proposition 2.33. One has a natural isomorophism

$$
D_{\eta, \psi, X} \cong D_{\psi+\sum_{Y \in S}} \eta(Y) \psi_{Y}, X .
$$

Proof. The required isomorphism is implemented locally near $x \in X$ by conjugation of twisted differential operators by the multivalued function $\prod_{Y \in S} z_{Y}^{\eta_{Y}}$, where $z_{Y}$ is a regular function on a neighborhood of $x$ such that the subscheme $Y \subset X$ is cut out in this neighborhood by the equation $z_{Y}=0$.

It will be convenient for us to make a similar modification of the sheaf $H_{t, c, \psi, X, G}$. Namely, let $\eta$ be a function on the set of conjugacy classes of $Y$ such that $(Y, g) \in S$. We define $H_{t, c, \eta, \psi, X, G}$ in the same way as $H_{t, c, \psi, X, G}$ except that the Dunkl-Opdam operators are defined by the formula

$$
D:=t \mathbf{L}_{v}+\sum_{(Y, g) \in S} f_{Y}(x)\left(\frac{2 c(Y, g)}{1-\lambda_{Y, g}}(g-1)+\eta(Y)\right) .
$$

The following generalization of Proposition 2.33 shows that this modification is, in fact, tautological.

Proposition 2.34. One has a natural isomorophism

$$
H_{t, c, \eta, \psi, X, G} \cong H_{t, c, \psi+\sum_{Y} \eta(Y) \psi_{Y}, X, G} .
$$

Proof. Since the terms in the Dunkl-Opdam operators containing $c$ are the same in both cases, it suffices to prove the statement for $c=0$. But this follows from Proposition 2.33 (and its classical limit).

Remark 2.35. We note that the restriction of the sheaf $H_{1, c, \eta, 0, X, G}$ to $X^{\circ} / G$, where $X^{\circ}:=X \backslash \cup_{(Y, g) \in S} Y$, is naturally isomorphic to $G \ltimes D_{X^{\circ}}$. Indeed, this follows from Proposition 2.15 and the fact that the line bundle $\mathcal{O}_{X}(Y)$ is trivial on $X^{\circ}$.

2.15. Examples. In this subsection we will give a few examples of Cherednik algebras and Calogero-Moser spaces of varieties. More details on these examples are given in the papers referenced in Section 4.

Example 2.36. Let $C$ be a smooth complex algebraic curve, and $X=C^{n}, G=S_{n}$ $(n>1)$. In this case, $c$ is a single parameter. If $C=\mathbb{C}$ then $H_{t, c, 0}(X, G)$ is the rational Cherednik algebra corresponding to the group $S_{n}$ acting in the permutation representation $\mathbb{C}^{n}$ (see $\mathrm{EG}$ ). If $C=\mathbb{C}^{*}$ then $H_{t, c, 0}(X, G)$ is the trigonometric Cherednik algebra for $S_{n}$ (the degenerate double affine Hecke algebra), defined by Cherednik; see e.g. the appendix to $\mathrm{BE}$. But for other curves we obtain new algebras. Note that if the curve is projective, then the sheaf $H_{t, c, 0, X, G}$ admits a 1-parameter deformation $H_{t, c, \psi, X, G}$ (where $\psi$ is a multiple of the class of the line bundle $L^{\bigotimes n}, L$ being a degree 1 line bundle on $C$ ).

Note that for any curve $C$ and $c \neq 0$, the varieties $M_{c, \psi}(X, G)$ are smooth and admit a Lagrangian projection to $S^{n} C$. This is checked by looking at formal neighborhoods of points of $X / G=S^{n} C$, where the statement follows from the results of [EG].

As an example, look at the case $C=\mathbb{P}^{1}$, and consider the algebra of global sections $A_{t, c, \omega}=\Gamma\left(H_{t, c, \omega, X, G}\right)$. It is easy to see that $A_{1,0, \psi}=S_{n} \ltimes D_{\psi}\left(\mathbb{P}^{1}\right)^{\otimes n}=$ $S_{n} \ltimes \mathcal{D}_{\psi}^{\otimes n}$, where $\mathcal{D}_{\psi}$ is the Dixmier quotient, i.e. the quotient of $U\left(\mathfrak{s l}_{2}\right)$ by the central character of an $\mathfrak{s l}_{2}$-module with highest weight $\psi$. More generally, let 
$H_{t, c^{\prime}}\left(\mathbb{C}^{n}, S_{n} \ltimes \mathbb{Z}_{2}^{n}\right), c^{\prime}=\left(c_{1}^{\prime}, c_{2}^{\prime}\right)$, be the rational Cherednik algebra of type $B_{n}$ (see [BEG1], Section 6). Let $\mathbf{p}$ be the averaging idempotent for the group $\mathbb{Z}_{2}^{n}$. Then one can show that $A_{t, c, \psi}$ is isomorphic to $\mathbf{p} H_{t, c^{\prime}}\left(\mathbb{C}^{n}, S_{n} \ltimes \mathbb{Z}_{2}^{n}\right) \mathbf{p}$ for $c^{\prime}$ related to $(c, \psi)$ by the invertible linear transformation: $c_{1}^{\prime}=c, c_{2}^{\prime}=\psi-(n-1) c+t / 2$.

This realization allows us to give a Borel-Weil type construction of some finite dimensional representations of $A_{1, c, \psi}=\mathbf{p} H_{1, c^{\prime}}\left(\mathbb{C}^{n}, S_{n} \ltimes \mathbb{Z}_{2}^{n}\right) \mathbf{p}$. Namely, assume that $t=1$ and $\psi=m$ is a nonnegative integer. Then the algebra $A_{1, c, \psi}$ admits a representation $W_{m}$ of dimension $(m+1)^{n}$, on global sections of the line bundle $\left(L^{\bigotimes n}\right)^{\otimes m}$. It can be checked that this representation has the form $\mathbf{p} N(\mathbb{C})$, where $N(\mathbb{C})$ is a highest weight representation of $H_{1, c^{\prime}}\left(\mathbb{C}^{n}, S_{n} \ltimes \mathbb{Z}_{2}^{n}\right)$ with trivial highest weight (of dimension $\left.(2 m+1)^{n}\right)$, introduced in [BEG1], Theorem 6.1.

Example 2.37. Let $\mathfrak{h}$ be a finite dimensional complex vector space, $G$ be a finite subgroup in $P G L(\mathfrak{h})$, and $G^{\prime}$ its preimage in $G L(\mathfrak{h})$. Let $\widehat{G}$ be the intersection of all subgroups of $G^{\prime}$ which project onto $G$, and which contain all complex reflections of $G^{\prime}$. It is easy to see that $\widehat{G}$ is a finite group. Let $K$ be the intersection of $\widehat{G}$ with the scalars in $G L(\mathfrak{h})$; it is clearly a cyclic group, and $\widehat{G} / K \subset G$.

Let $X=\mathbb{P h}$ be the projective space of $\mathfrak{h}$. Then we can define the sheaf $H_{t, c, \psi, X, G}$, where $\psi$ is a single parameter (corresponding to twisting by a power of the tautological line bundle).

Observe that the set $S$ of pairs $(Y, g)$ can be $G$-equivariantly identified with the set of complex reflections in $\widehat{G}$; thus the parameter $c$ is a function on the set of conjugacy classes of complex reflections in $\widehat{G}$.

Consider the algebra of global sections of the sheaf $H_{t, c, \psi, X, G}$, which we denote by $A_{t, c, \psi}$. It is easy to show that $A_{1,0, \psi}=G \ltimes D_{\psi}(\mathbb{P h})$. In other words, $A_{1,0, \psi}=$ $(\widehat{G} \ltimes D(\mathfrak{h})[0]) /(E=\psi, K=1)$, where $D(\mathfrak{h})[0]$ is the algebra of differential operators on $\mathfrak{h}$ which commute with the Euler vector field $E$. More generally, one can show (see [BM], Lemma 5.4.1) that

$$
A_{t, c, \psi}=H_{t, c}(\mathfrak{h}, \widehat{G})[0] /\left(\mathbf{h}=\frac{\ell t}{2}-\sum_{s \in S} \frac{2 c_{s}}{1-\lambda_{s}}+\psi, K=1\right),
$$

(note that $\mathbf{h}$ is a central element of $H_{t, c}(\mathfrak{h}, G)[0]$ ).

Example 2.38. Let us specialize the previous example to the case $\operatorname{dim} \mathfrak{h}=2$. Thus $G \subset P S L(2, \mathbb{C})$. Let $\Gamma$ be the preimage of $G$ in $S L(2, \mathbb{C})$. The kernel of the map from $\Gamma$ to $G$ consists of the identity 1 and minus identity $z$.

Note that we have a natural bijection from $S L(2, \mathbb{C}) \backslash\{1, z\}$ to the set of pairs $(y, g)$, where $g \in P S L(2, \mathbb{C})$ and $y \in \mathbb{P}^{1}$ is a fixed point of $g$. This map is defined by $\gamma \mapsto(y, \bar{\gamma})$, where $\bar{\gamma}$ is the projection of $\gamma$ to $P S L(2, \mathbb{C})$, and $y$ is the fixed line of $\gamma$ on which it acts with eigenvalue having positive imaginary part. This shows that the set $S$ can be identified with the set of conjugacy classes in $\Gamma$ of elements not equal to $1, z$. Let $\mathbf{p}_{z}=(1+z) / 2$.

Consider the sheaf $H_{t, c, \psi}\left(\mathbb{P}^{1}, G\right)$. Let the algebra of its global sections be denoted by $A_{t, c, \psi}$. It is easy to show that $A_{1,0, \psi}=G \ltimes D_{\psi}\left(\mathbb{P}^{1}\right)$.

Recall that in $\mathrm{CBH}$, Crawley-Boevey and Holland defined a family of algebras $Q_{\lambda}$, parametrized by elements $\lambda$ of the center of $\mathbb{C}[\Gamma]$, which are the quotient of the smash product $\Gamma \ltimes T\left(\mathbb{C}^{2}\right)$ of $\Gamma$ with the tensor algebra of the tautological representation by the ideal generated by $x y-y x-\lambda$ (where $x, y$ is a basis of $\mathbb{C}^{2}$ ). 
Thus, $A_{1,0, \psi}=\mathbf{p}_{z} Q_{\lambda} \mathbf{p}_{z}$ for $\lambda=1-z$. More generally, one can show that $A_{t, c, \psi}=\mathbf{p}_{z} Q_{\lambda} \mathbf{p}_{z}$, where $\lambda$ is related by an invertible transformation with $(t, c, \psi)$ (see [FT]).

The combination of the above two examples shows that the algebra $\mathbf{p}_{z} Q_{\lambda} \mathbf{p}_{z}$ can be obtained as a quotient of $H_{t, c}(\mathfrak{h}, \widehat{G})[0]$.

This allows one to explicitly construct finite dimensional representations of $\mathbf{p}_{z} Q_{\lambda} \mathbf{p}_{z}$ (this is done somewhat implicitly in $[\mathrm{CBH}]$ ). One way is to look at representations of $H_{t, c}(\mathfrak{h}, W)[0]$ on weight subspaces of representations of $H_{t, c}(\mathfrak{h}, W)$ from category $\mathcal{O}_{c / t}(\mathfrak{h}, W)$ (for $t \neq 0$ ). Another method is to use the isomorphism $A_{t, c, \psi} \cong \mathbf{p}_{z} Q_{\lambda} \mathbf{p}_{z}$, and consider finite dimensional representations of $A_{t, c, \psi}$ for integer $\psi=m$ on sections of the $G$-equivariant vector bundle $L \otimes V$ on $\mathbb{P}^{1}$ (where $L$ is the tautological line bundle, and $V$ is an irreducible representation of $\Gamma$ in which $z$ acts by $\left.(-1)^{m}\right)$.

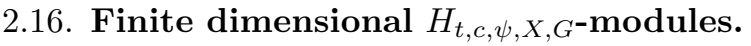

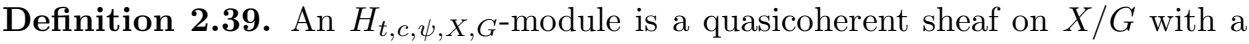
compatible action of the sheaf of algebras $H_{t, c, \psi, X, G}$.

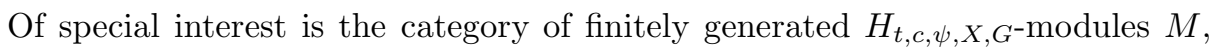
i.e., quotients of $H_{t, c, \psi, X, G}^{\oplus n}$. This category is a generalization of the category of finitely generated $G$-equivariant twisted D-modules on $X$, and deserves a special study, but we will limit ourselves to discussing finite dimensional objects $M$ in this category, i.e., such that the space of sections $\Gamma(U, M)$ on every open set $U$ is finite dimensional. Such modules exist for $t=0$ and also for $t \neq 0$ and special values of $c$.

For $x \in X$, let $G_{x}$ be the stabilizer of $x$ in $G$; then $G_{x} \subset G L\left(T_{x} X\right)$. Let $c_{x}$ be the function on the set of conjugacy classes of complex reflections in $G_{x}$ defined by $c_{x}(g)=c\left(Y_{g}, g\right)$, where $Y_{g}$ is the component of $X^{g}$ that passes through $x$.

Proposition 2.40. (i) Let $M$ be an indecomposable finite dimensional module over $H_{t, c, \psi, X, G}$. Then the set-theoretical support of $M$ on $X / G$ consists of one point $z \in X / G$.

(ii) Let $x$ be any point of $X$ projecting to $z$. The category of finite dimensional

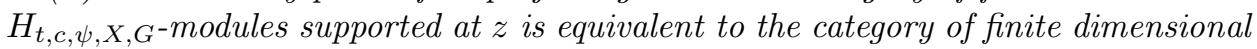
modules over the rational Cherednik algebra $H_{t, c_{x}}\left(T_{x} X, G_{x}\right)$ attached to the group $G_{x}$ acting in $T_{x} X$.

Proof. (i) The statement follows from the fact that the adjoint action of $\mathcal{O}_{X / G}(U)$ on $H_{t, c, \psi, X, G}\left(U^{\prime}\right)$ is locally nilpotent.

(ii) Let $M$ be a finite dimensional $H_{t, c, \psi, X, G}$ module supported at $z$. Then the maximal ideal sheaf $I_{z}$ of $z$ acts nilpotently on $M$, so $M$ can be extended to a module over the degree-wise completion $\widehat{H}_{t, c, \psi, X, G, z}$ of $H_{t, c, \psi, X, G}$ with respect to $I_{z}$ (see Subsection 2.7).

Let $\phi: X \rightarrow X / G$ be the natural map. By Proposition [2.16, the algebra $\widehat{H}_{t, c, \psi, X, G, z}$ is isomorphic (non-canonically) to $\oplus_{y \in \phi^{-1}(z)} \widehat{H}_{t, c_{y}}\left(T_{y} X, G_{y}\right)$, where hat denotes the completion defined in Subsection 2.6. Thus the fiber $M_{x}$ of $M$ over $x$ (as an $\mathcal{O}$-module) has a natural structure of a $\widehat{H}_{t, c_{x}}\left(T_{x} X, G_{x}\right)$-module. Restricting this module to the algebra $H_{t, c}\left(T_{x} X, G_{x}\right)$, we obtain a functor in one direction, given by $M \mapsto M_{x}$.

To construct the functor in the opposite direction, let $N$ be a finite dimensional module over $H_{t, c_{x}}\left(T_{x} X, G_{x}\right)$. Let $N^{\prime}=\operatorname{Ind}_{G_{x}}^{G}(N)$. Arguing as above, we can turn 
$N^{\prime}$ into a module over $H_{t, c, \psi, X, G}$. So the desired functor is $N \mapsto N^{\prime}$. It is easy to verify that the two functors are mutually inverse, which proves (ii).

Thus, the problem of describing finite dimensional representations of $H_{t, c, \psi, X, G}$ reduces to the linear case, which is treated in BEG1] and references therein.

2.17. Quasiinvariants. In this subsection we will discuss the global version of the theory of quasiinvariants for reflection groups. One can generalize it to the complex reflection case using the ideas of $[\mathrm{BC}$.

Let $X$ be a smooth complex algebraic variety, $G \subset \operatorname{Aut}(X)$, and assume that the quotient $X / G$ exists as a variety. Let $S_{2} \subset S$ be the set of pairs $(Y, g) \subset S$ for which $g^{2}=1$. Any function on $S_{2}$ can be regarded as a function on $S$ using extension by zero.

Recall that $\phi: X \rightarrow X / G$ denotes the natural map.

Let $m: S_{2} \rightarrow \mathbb{Z}_{+}$be a conjugation invariant function. The sheaf of $m$-quasiinvariants of $X, G$, denoted $Q_{m, X, G}$, is defined to be the subsheaf of $\phi_{*} \mathcal{O}_{X}$ whose local sections satisfy the condition: for any $(Y, g) \in S_{2}, f-{ }^{g} f$ vanishes to order $2 m(Y, g)+1$ at $Y$. This is a sheaf of rings on $X / G$. This sheaf is the structure sheaf of an algebraic variety $X_{m}$, together with a natural map $\zeta: X \rightarrow X_{m}$ (the normalization map). By looking at formal neighborhoods of points, it is easy to check (see [BEG]) that $\zeta$ is birational and bijective.

With these definitions, it is not hard to see that the main results of [EG1, BEG] extend to the global case. Namely, we have the following result.

Proposition 2.41. $X_{m}$ is a Gorenstein variety.

Proof. The statement is local with respect to $X / G$, so it can be checked on formal neighborhoods of points, which is the Feigin-Veselov conjecture proved in EG1, BEG.

Now observe that the sheaf of algebras $H_{1, m, 0, X, G}$ acts on $\phi_{*} \mathcal{O}_{X}$. Hence the sheaf of spherical subalgebras e $H_{1, m, 0, X, G}$ e acts in e $\phi_{*} \mathcal{O}_{X}=\mathcal{O}_{X / G}$ by differential operators. Thus we can extend this action to an action on $\mathbb{C}(X)$ (by using the same differential operators).

Proposition 2.42. $Q_{m, X, G}$ is invariant under the action of the sheaf of spherical subalgebras $\mathrm{e} H_{1, m, 0, X, G} \mathrm{e}$.

Proof. This is again a local statement, so it can be checked on formal neighborhoods of points, which is done in [BEG].

\section{Cherednik Algebras of Analytic varieties and Hecke Algebras}

The construction and main properties of the Cherednik algebras of algebraic varieties can be extended without significant changes to the case when $X$ is a complex analytic variety. We will not specify the routine modifications involved; rather, we will use Cherednik algebras for analytic varieties to define certain deformations of orbifold fundamental groups which we call Hecke algebras, and prove that in certain cases they are flat. 
3.1. Orbifold fundamental group. Let $X$ be a connected complex analytic variety, and $G$ is a finite group of automorphisms of $X$. Then $X / G$ is a complex orbifold (for basics on orbifolds, see Da]). Let $x \in X$ be a point with trivial stabilizer. In this case we can define the orbifold fundamental group $\pi_{1}^{\text {orb }}(X / G, x)$, [Da]. This group is generated by homotopy classes of paths on $X$ connecting $x$ and $g x$ for $g \in G$, with multiplication defined by the rule: $\gamma_{1} \circ \gamma_{2}$ is $\gamma_{2}$ followed by $g \gamma_{1}$, where $g$ is such that $g x$ is the endpoint of $\gamma_{2}$. We have an exact sequence

$$
1 \rightarrow \pi_{1}(X, x) \rightarrow \pi_{1}^{\text {orb }}(X / G, x) \rightarrow G \rightarrow 1 .
$$

Note also that $\pi_{1}^{\text {orb }}(X / G, x)=\pi_{1}((X \times E G) / G, x)$, where $E G$ is the universal cover of the classifying space of $G$. In other words, the action of $G$ on $X$ gives rise to an associated bundle with fiber $X$ over the classifying space $B G$, and $\pi_{1}^{\text {orb }}(X / G, x)$ is the fundamental group of the total space of this bundle.

Let $Z$ be the set of all points of $X$ having a nontrivial stabilizer; then $Z$ is a closed subset of $X$. Let $X^{\prime}=X \backslash Z$.

Definition 3.1. The fundamental group $\pi_{1}\left(X^{\prime} / G, x\right)$ will be called the braid group of $X / G$.

Now let $S$ be the set of pairs $(Y, g)$ such that $g \neq 1$ and $Y \subset X^{g}$ is a reflection hypersurface. For $(Y, g) \in S$, let $G_{Y}$ be the subgroup of $G$ whose elements act trivially on $Y$. This group is obviously cyclic; let $n_{Y}=\left|G_{Y}\right|$. Let $C_{Y}$ be the conjugacy class in $\pi_{1}\left(X^{\prime} / G, x\right)$ corresponding to a small circle going counterclockwise around the image of $Y$ in $X / G$.

Proposition 3.2. The group $\pi_{1}^{\mathrm{orb}}(X / G, x)$ is the quotient of the braid group $\pi_{1}\left(X^{\prime} / G, x\right)$ by the relations $T^{n_{Y}}=1$ for all $T \in C_{Y}$.

Proof. We have a natural surjective map $\theta: \pi_{1}\left(X^{\prime} / G, x\right) \rightarrow \pi_{1}^{\text {orb }}(X / G, x)$ induced by the embedding $X^{\prime} \rightarrow X$. The kernel of this map obviously contains the elements $T^{n_{Y}}, T \in C_{Y}$. The fact that the kernel is generated by (conjugates of) these elements follows from the Seifert-van Kampen theorem.

3.2. The Hecke algebra of $X, G$. For any conjugacy class of hypersurfaces $Y$ such that $(Y, g) \in S$ we introduce formal parameters $\tau_{1 Y}, \ldots, \tau_{n_{Y} Y}$. The entire collection of these parameters will be denoted by $\tau$.

Definition 3.3. We define the Hecke algebra of $X, G$, denoted $\mathcal{H}_{\tau}(X, G, x)$, to be the quotient of the $\tau$-adically completed group algebra of the braid group, $\mathbb{C}\left[\pi_{1}\left(X^{\prime} / G, x\right)\right][[\tau]]$, by the $\tau$-adically closed ideal defined by the relations

$$
\prod_{j=1}^{n_{Y}}\left(T-e^{2 \pi i j / n_{Y}} e^{\tau_{j Y}}\right)=0, T \in C_{Y} .
$$

It is clear that up to an isomorphism this algebra is independent on the choice of $x$, so we will sometimes drop $x$ form the notation. It follows from Proposition 3.2 that $\mathcal{H}_{\tau}(X, G) /(\tau=0)=\mathbb{C}\left[\pi_{1}^{\text {orb }}(X / G)\right]$. Thus, $\mathcal{H}_{\tau}(X, G)$ is a deformation of $\mathbb{C}\left[\pi_{1}^{\text {orb }}(X / G)\right]$.

Remark 3.4. One can also define the Hecke algebra $\mathcal{H}_{\tau}(X, G)$ for complex parameters $\tau_{j Y}$ (or, rather, $q_{j Y}=e^{\tau_{j Y}}$ ), generalizing the above formal definition in an obvious way. 
3.3. The Knizhnik-Zamolodchikov functor. In this subsection we will define a global analog of the KZ functor defined in [GGOR.

As we mentioned, similarly to the algebraic case we can define the sheaf of algebras $H_{1, c, \eta, 0, X, G}$ on $X / G$ (in the analytic topology). Note that the restriction of this sheaf to $X^{\prime} / G$ is the same as the restriction of the sheaf $G \ltimes D_{X}$ to $X^{\prime} / G$ (i.e. on $X^{\prime} / G$, the dependence of the sheaf on the parameters $c$ and $\eta$ disappears). This follows from the fact that the line bundles $\mathcal{O}_{X}(Y)$ become trivial when restricted to $X^{\prime}$.

Now let $M$ be a module over $H_{1, c, \eta, 0, X, G}$ which is a locally free coherent sheaf when restricted to $X^{\prime} / G$. Then the restriction of $M$ to $X^{\prime} / G$ is a $G$-equivariant D-module on $X^{\prime}$ which is coherent and locally free as an $\mathcal{O}$-module. Thus, $M$ corresponds to a locally constant sheaf (local system) on $X^{\prime} / G$, which gives rise to a monodromy representation of the braid group $\pi_{1}\left(X^{\prime} / G, x\right)$ on the fiber $M_{x}$ of $M$ at $x$. This representation will be denoted by $K Z(M)$. This defines a functor KZ, which is analogous to the one in GGOR.

It follows from the theory of D-modules that any $\mathcal{O}_{X / G^{-}}$coherent $H_{1, c, \eta, 0, X, G^{-}}$ module is locally free when restricted to $X^{\prime} / G$. Thus the $\mathrm{KZ}$ functor acts from the abelian category $\mathcal{C}_{c, \eta}$ of $\mathcal{O}_{X / G^{-}}$-coherent $H_{1, c, \eta, 0, X, G^{-m o d u l e s ~ t o ~ t h e ~ c a t e g o r y ~ o f ~}}$ finite dimensional representations of $\pi_{1}\left(X^{\prime} / G, x\right)$. It is easy to see that this functor is exact.

For any reflection hypersurface $Y$, let $g_{Y}$ be the generator of $G_{Y}$ which has eigenvalue $e^{2 \pi i / n_{Y}}$ in the normal bundle to $Y$. Let $(c, \eta) \mapsto \tau(c, \eta)$ be the invertible linear transformation defined by the formula

$$
\tau_{j Y}=-\frac{2 \pi i}{n_{Y}}\left(2 \sum_{m=1}^{n_{Y}-1} c\left(Y, g_{Y}^{m}\right) \frac{1-e^{-2 \pi i j m / n_{Y}}}{1-e^{-2 \pi i m / n_{Y}}}+\eta(Y)\right) .
$$

Proposition 3.5. The functor $K Z$ maps the category $\mathcal{C}_{c, \eta}$ to the category of representations of the algebra $\mathcal{H}_{\tau(c, \eta)}(X, G)$.

Proof. Let $Y$ be a reflection hypersurface. Our job is to show that for any $M \in \mathcal{C}_{c, \eta}$, every $T \in C_{Y}$ satisfies the Hecke relation (4) on $K Z(M)$.

Let $y \in Y$ be a generic point. Using Lemma 2.8(i), we may reduce the problem to the special case when $X$ is a 1-dimensional open disk $B$ centered at $0, Y=\{0\}$, and $G=G_{Y}=\mathbb{Z} / n \mathbb{Z}$ acts by rotations. We may also assume without loss of generality that $\eta=0$, since the algebra $H_{1, c, \eta}(B, \mathbb{Z} / n \mathbb{Z})$ may be identified with $H_{1, c, 0}(B, \mathbb{Z} / n \mathbb{Z})$ by conjugation by $z^{\eta}$, where $z$ is the coordinate on $B$, and this results in rescaling $T$ by $e^{2 \pi i \eta / n}$.

Let $M \in \mathcal{C}_{c, 0}$, and let $\widehat{M}:=\mathbb{C}[[z]] \otimes_{\mathcal{O}(B)} M$ be the restriction of $M$ to the formal neighborhood of zero (where $\mathcal{O}(B)$ is the algebra of holomorphic functions on $B$ ). Then $\widehat{M}$ is a module over $\widehat{H}_{1, c}(\mathbb{C}, \mathbb{Z} / n \mathbb{Z})$, which is finitely generated over $\mathbb{C}[[z]]$. By Proposition 2.9, there exists a unique $M_{0} \in \mathcal{O}_{c}(\mathbb{C}, \mathbb{Z} / n \mathbb{Z})$ such that $\widehat{M} \cong \widehat{M}_{0}$, and it is not hard to show that $M \cong \mathcal{O}(B) \otimes_{\mathbb{C}[z]} M_{0}$ as a $H_{1, c, 0}(B, \mathbb{Z} / n \mathbb{Z}$ )-module (e.g., for $G=1$ this is just the well known statement that any $\mathcal{O}$-coherent $D$-module on $B$ is a multiple of $\mathcal{O}$ ). Therefore, we may replace $B$ with $\mathbb{C}$ and $M$ with $M_{0}$.

Now recall GGOR, Theorem 5.13:

Theorem. If $G \subset G L(\mathfrak{h})$ is a complex reflection group, and $N$ is a module over $H_{1, c}(\mathfrak{h}, G)$ from category $\mathcal{O}_{c}(\mathfrak{h}, G)$ then $K Z(N)$ is a module over $\mathcal{H}_{\tau(c, 0)}(\mathfrak{h}, G)$. 
Thus, the required statement follows from [GGOR, Theorem 5.13 for cyclic groups $G$.

Remark 3.6. Note that Theorem 5.13 of GGOR for cyclic groups $G$ is very easy to prove. Namely, let $n=|G|$, and $g$ be the generator of $G$ whose nontrivial eigenvalue in $\mathfrak{h}$ is $e^{2 \pi i / n}$. Let $\chi_{j}$ be the character of $G$ given by $\chi_{j}(g)=e^{2 \pi i j / n}$. An easy computation (see [GGOR] ) shows that if $N$ is the standard (=Verma) module $M\left(\chi_{j}\right)$ with highest weight $\chi_{j}$, then $K Z(N)$ is the 1-dimensional character $\zeta_{j}$ of $\mathcal{H}_{\tau}$, given by

$$
\zeta_{j}(T)=\exp \left(\frac{2 \pi i}{n}\left(j-2 \sum_{m=1}^{n-1} c_{g^{m}} \frac{1-e^{-2 \pi i j m / n}}{1-e^{-2 \pi i m / n}}\right)\right) .
$$

This implies the statement for regular $c$, for which the category $\mathcal{O}_{c}$ is semisimple. In general, since $K Z$ is an exact functor, it suffices to prove the statement for projective objects $N=P$. But a projective object $P$ admits a flat deformation to regular values of $c$, so the statement follows from the regular case by taking a limit.

3.4. The flatness theorem. The main result of this section is the following theorem.

Theorem 3.7. Assume that $\pi_{2}(X) \otimes \mathbb{Q}=0$. Then $\mathcal{H}_{\tau}(X, G)$ is a flat formal deformation of $\mathbb{C}\left[\pi_{1}^{\text {orb }}(X / G)\right]$.

The rest of the section contains the proof of Theorem 3.7 and examples of its application.

3.5. A lemma on deformations. We keep the conventions of Subsections 3.1 , 3.2. Let $\widetilde{X}$ be the universal covering space of $X$ with base point $x$. Let $\pi: \widetilde{X} \rightarrow X$ be the covering map. Consider the sheaf $\pi_{!} \mathcal{O}_{\tilde{X}}$, the direct image with compact supports of the structure sheaf. Namely, for a small ball $U \subset X, \Gamma\left(U, \pi_{!} \mathcal{O}_{\tilde{X}}\right)$ is the space of analytic functions on $\pi^{-1}(U)$ supported on finitely many connected components of $\pi^{-1}(U)$. This sheaf has a natural structure of a D-module on $X$ (in general, not coherent). Let $M=\operatorname{Ind}_{D_{X}}^{G \ltimes D_{X}} \pi_{!} \mathcal{O}_{\widetilde{X}}$ be the corresponding equivariant $D$-module. Thus $M$ is a module over $H_{1,0,0,0, X, G}$.

A central role in the proof of Theorem 3.7 is played by the following lemma.

Lemma 3.8. If $\pi_{2}(X) \otimes \mathbb{Q}=0$ then the $G$-equivariant $D$-module $M$ has a unique flat formal deformation to a module over $H_{1, c, \eta, \psi, X, G}$.

Proof. As usual, classes of first order deformations of $M$ lie in $\operatorname{Ext}^{1}(M, M)$ and obstructions in $\operatorname{Ext}^{2}(M, M)$ (where the Exts are taken in the category of $G$-equivariant $D$-modules on $X)$. So it suffices to show that these two Ext groups vanish.

Using Shapiro's lemma and the fact that the functor $\pi^{*}$ (sheaf-theoretic inverse image) is right adjoint to $\pi$, we have

$$
\operatorname{Ext}_{G \ltimes D_{X}}^{i}(M, M)=\operatorname{Ext}_{D_{X}}^{i}\left(\pi_{!} \mathcal{O}_{\widetilde{X}}, \operatorname{Res} M\right)=\operatorname{Ext}_{D_{\widetilde{X}}}^{i}\left(\mathcal{O}_{\widetilde{X}}, \pi^{*} \operatorname{Res} M\right) .
$$

(Here Res denotes the functor of forgetting the $G$-equivariant structure of a $G$ equivariant D-module on $X)$. But it is clear that $\pi^{*} \operatorname{Res} M=\mathcal{O}_{\tilde{X}} \otimes \mathbb{C}\left[\pi_{1}^{\text {orb }}(X / G)\right]$. Thus,

$$
\operatorname{Ext}_{G \ltimes D_{X}}^{i}(M, M)=\operatorname{Ext}_{D_{\widetilde{X}}}^{i}\left(\mathcal{O}_{\widetilde{X}}, \mathcal{O}_{\widetilde{X}}\right) \otimes \mathbb{C}\left[\pi_{1}^{\text {orb }}(X / G)\right]
$$


Thus, by Remark 2.2, we finally obtain

$$
\operatorname{Ext}_{G \ltimes D_{X}}^{i}(M, M)=H^{i}(\widetilde{X}, \mathbb{C}) \otimes \mathbb{C}\left[\pi_{1}^{\text {orb }}(X / G)\right] .
$$

Since $\widetilde{X}$ is simply connected, this clearly vanishes for $i=1$. Now consider the case $i=2$. Since $\pi_{2}(X) \otimes \mathbb{Q}=0$, we have $\pi_{2}(\tilde{X}) \otimes \mathbb{Q}=0\left(\right.$ as $\left.\pi_{2}(X)=\pi_{2}(\widetilde{X})\right)$, hence by Hurewicz's theorem $H^{2}(\widetilde{X}, \mathbb{C})=0$. Thus Ext ${ }^{2}$ also vanishes, and we are done.

3.6. Proof of Theorem 3.7, Let $M_{c, \eta, \psi}$ be the flat formal deformation of $M$ whose existence and uniqueness is claimed in Lemma 3.8. Then $M_{c, \eta}:=M_{c, \eta, 0}$ becomes an ordinary $G$-equivariant D-module when restricted to the open set $X^{\prime}$.

We begin with explaining that even though the module $M_{c, \eta}$ is, in general, infinitely generated as an $\mathcal{O}$-module (as the cover $\pi$ may have infinitely many sheets), we can still apply the functor $K Z$ to it and obtain a braid group representation $K Z\left(M_{c, \eta}\right)$. Essentially, this is possible because the parameters $c, \eta$ are formal, and thus the differential equation whose monodromy needs to be computed can be solved using Chen integrals, like in Drinfeld's work on the formal KZ equation Dr.

In more detail, let $\left\{B_{i}\right\}$ be a cover of $X^{\prime} / G$ by small balls. On each $B_{i}$, the module $M$ can be trivialized, i.e., identified with $\mathcal{O} \otimes F$, where $F=\mathbb{C}\left[\pi_{1}^{\text {orb }}(X / G)\right]$, so that the flat connection on $\left.M\right|_{X^{\prime} / G}$ becomes the trivial connection. Then we get transition maps $g_{i j}^{0}$ on $B_{i} \cap B_{j}$, which are just elements of $\pi_{1}^{\text {orb }}(X / G)$.

Now consider the module $M_{c, \eta}$, which is a deformation of $M_{0,0}=M$. On $X^{\prime} / G$, this is a deformation of (possibly infinite dimensional) bundles with a flat connection, so it can be understood as a collection of elements $g_{i j}(c, \eta)(z)$ (transition functions), and $\omega_{i}(c, \eta)$ (connection forms). Here $g_{i j}(c, \eta)$ are formal series in $c, \eta$ with coefficients in $\operatorname{Hom}_{\mathbb{C}}\left(F, \mathcal{O}\left(B_{i} \cap B_{j}\right) \otimes F\right)$, and $\omega_{i}(c, \eta)$ are formal series in $c, \eta$ with coefficients in $\operatorname{Hom}_{\mathbb{C}}\left(F, \Omega^{1, c l}\left(B_{i}\right) \otimes F\right)$, such that $g_{i j}(c, \eta)$ satisfy the cocycle condition for transition functions, the connections $d+\omega_{i}(c, \eta)$ on $B_{i}$ glue into a flat connection on $X^{\prime} / G$, and $g_{i j}(0,0)=g_{i j}^{0}, \omega_{i}(0,0)=0$. Now, given a path $\gamma$ starting and ending at $x$, it can be subdivided into finitely many consecutive segments $\gamma_{k}$, $k=1, \ldots, n$, each contained in a single ball $B_{i_{k}}$. Here $x$ is the beginning of $\gamma_{1}$ and the end of $\gamma_{n}$, and for convenience we use the same $i_{1}=i_{n}$ for all $\gamma$. Solving the differential equation $d f+\omega_{i_{k}}(c, \eta) f=0$ in $B_{i_{k}}$ (which we can do using Chen integrals since $\omega(0,0)=0$, even though $\operatorname{dim} F$ may be infinite), we find the monodromy operator $A_{k}$ from the beginning to the end of $\gamma_{k}$. Clearly, $A_{k} \in 1+I \operatorname{End} F$, where $I$ is the maximal ideal in $\mathbb{C}[[c, \eta]]$. The monodromy operator $A(\gamma)$ along $\gamma$ is then defined to be $A_{\gamma}=A_{n} g_{i_{n}, i_{n-1}} A_{n-1} \ldots A_{2} g_{i_{2} i_{1}} A_{1}$. Then $\gamma \mapsto A_{\gamma}$ defines a representation of the braid group on $F[[c, \eta]]$. This is the desired monodromy representation $K Z\left(M_{c, \eta}\right)$.

Next, we claim that $K Z\left(M_{c, \eta}\right)$ is, in fact, a representation of the Hecke algebra $\mathcal{H}_{\tau(c, \eta)}(X, G)$; in other words, we can apply Proposition 3.5 even though the representation may be infinite dimensional.

To see this, fix a reflection hypersurface $Y$ and a generic point $y \in Y$. Now use Lemma[2.8(i) to replace $X$ with a small ball $B$ around $y$ (so that $B$ is invariant under the stabilizer $G_{Y}$ and transversal to $Y$ ), and $G$ with $G_{Y}$. We have a (non-canonical) isomorphism $\left.M\right|_{B}=W \otimes M^{B}$, where $M^{B}$ is the analog of the module $M$ for $X$ replaced with $B$ and $G$ replaced with $G_{Y}$, and $W$ is a vector space. Namely, $W$ may be identified with the space $\mathbb{C}\left[\pi_{1}^{\text {orb }}(X / G) / G_{Y}\right]$ of finitely supported functions on the homogeneous space $\pi_{1}^{\text {orb }}(X / G) / G_{Y}$ (here we assume that the base point $x$ is contained in $B$ ). By Lemma 3.8, the unique deformation of $M^{B}$ to a module 
over $H_{1, c_{Y}, \eta_{Y}, 0, B, G_{Y}}$ (where $c_{Y}, \eta_{Y}$ are appropriate restrictions of $c, \eta$ ) is $M_{c_{Y}, \eta_{Y}}^{B}$. This implies that $\left.M_{c, \eta}\right|_{B}$ is isomorphic to the $\left(\left(c_{Y}, \eta_{Y}\right)\right.$-adically completed $)$ tensor product $W \otimes M_{c_{Y}, \eta_{Y}}^{B}$. But $M^{B}$ has finite dimensional fibers (their dimension is the order $n_{Y}$ of $G_{Y}$ ), so Proposition 3.5 can be applied to $M^{B}$. Thus we get that $K Z\left(\left.M_{c, \eta}\right|_{B}\right)$ is a representation of the Hecke algebra of $B$ (i.e., the monodromy operator around the center of $B$ satisfies the Hecke relation). Since this is valid for all $Y$, we see that $K Z\left(M_{c, \eta}\right)$ is a representation of the Hecke algebra $\mathcal{H}_{\tau(c, \eta)}(X, G)$, as desired.

Now we are ready to finish the proof of Theorem 3.7. As we have shown, the braid group representation $K Z\left(M_{c, \eta}\right)$ factors through the Hecke algebra $\mathcal{H}_{\tau(c, \eta)}(X, G)$. If $c, \eta=0$, this representation is the regular representation of the orbifold fundamental group $\pi_{1}^{\text {orb }}(X / G)$. Thus, the regular representation of $\pi_{1}^{\text {orb }}(X / G)$ admits a flat deformation to a representation of the Hecke algebra $\mathcal{H}_{\tau(c, \eta)}(X, G)$, implying that the Hecke algebra is flat. The theorem is proved.

3.7. Examples. In this subsection we would like to discuss a few examples of Hecke algebras and of application of Theorem 3.7

Example 3.9. Let $\mathfrak{h}$ be a finite dimensional vector space, and $W$ be a complex reflection group in $G L(\mathfrak{h})$. Then $\mathcal{H}_{\tau}(\mathfrak{h}, W)$ is the Hecke algebra of $W$ studied in BMR. It follows from Theorem 3.7 that this Hecke algebra is flat. This proof of flatness is, in fact, essentially the same as the original proof of this result given in BMR (based on the Dunkl-Opdam-Cherednik operators).

Example 3.10. Let $T$ be a maximal torus of a simply connected complex simple Lie group $G$, and $W=W(T)$ be its Weyl group. Then $\mathcal{H}_{\tau}(T, W)$ is the affine Hecke algebra. This algebra is also flat by Theorem 3.7. In fact, its flatness is a well known result from representation theory; our proof of flatness is essentially due to Cherednik [Ch].

Example 3.11. Let $W, T$ be as in the previous example, and $Q^{\vee}$ be the dual root lattice of $G$. Let $E$ be an elliptic curve, and $X=E \otimes Q^{\vee}$ be the corresponding Looijenga variety. Then $H_{\tau}(X, W)$ is the double affine Hecke algebra of Cherednik (Ch), and it is flat by Theorem 3.7. The fact that this algebra is flat was proved by Cherednik, Sahi, Noumi, Stokman (see [Ch], $[\mathrm{Sa}]$, [NoSt], $[\mathrm{St}]$ ) using a different approach (q-deformed Dunkl operators).

Example 3.12. Let $H$ be a simply connected complex Riemann surface (i.e., Riemann sphere $\mathbb{C P}^{1}$, the Euclidean plane $\mathbb{C}$, or the Lobachevsky plane $\mathbb{C}_{+}$), and $\Gamma$ be a cocompact lattice in $\operatorname{Aut}(H)$. Let $\Sigma=H / \Gamma$. Then $\Sigma$ is a compact complex Riemann surface. When $\Gamma$ contains elliptic elements (i.e., nontrivial elements of finite order), we are going to regard $\Sigma$ as an orbifold: it has special points $P_{i}, i=1, \ldots, m$ with stabilizers $\mathbb{Z}_{n_{i}}$. Then $\Gamma$ is the orbifold fundamental group of $\Sigma$.

Let $g$ be the genus of $\Sigma$, and $a_{i}, b_{i}, i=1, \ldots, g$, be the a-cycles and b-cycles of $\Sigma$. Let $c_{j}$ be the counterclockwise loops around $P_{j}$. Then $\Gamma$ is generated by $a_{l}, b_{l}, c_{j}$ with relations

$$
c_{j}^{n_{j}}=1, c_{1} c_{2} \ldots c_{m}=\prod_{l} a_{l} b_{l} a_{l}^{-1} b_{l}^{-1}
$$

For each $j$, introduce formal parameters $\tau_{k j}, k=1, \ldots, n_{j}$. Define the Hecke algebra $\mathcal{H}_{\tau}(\Sigma)$ of $\Sigma$ to be generated over $\mathbb{C}[[\tau]]$ by the same generators $a_{l}, b_{l}, c_{j}$ with defining 
relations

$$
\prod_{k=1}^{n_{j}}\left(c_{j}-e^{2 \pi i j / n_{j}} e^{\tau_{k j}}\right)=0, c_{1} c_{2} \ldots c_{m}=\prod_{l} a_{l} b_{l} a_{l}^{-1} b_{l}^{-1} .
$$

Thus $\mathcal{H}_{\tau}(\Sigma)$ is a deformation of $\mathbb{C}[\Gamma]$.

We claim that this deformation is flat if $H=\mathbb{C}$ or $H=\mathbb{C}_{+}$. To show this, let $\Gamma^{\prime}$ be a normal subgroup of $\Gamma$ of finite index acting freely on $H$. Let $G=\Gamma / \Gamma^{\prime}$, and $X=H / \Gamma^{\prime}$. Then $\mathcal{H}_{\tau}(\Sigma)=\mathcal{H}_{\tau}(X, G)$, so the result follows from Theorem 3.7 and the fact that $\pi_{2}(X)=\pi_{2}(H)=0$.

Remark 3.13. We note that if $H=\mathbb{C P}^{1}$ (so that the condition $\pi_{2}(X) \otimes \mathbb{Q}=0$ is violated) and $\Gamma \neq 1$, then this deformation is not flat, which shows that the assumption $\pi_{2}(X) \otimes \mathbb{Q}$ in Theorem 3.7 cannot be removed. Indeed, let $\tau=\tau(\hbar)$ be a 1-parameter subdeformation of $\mathcal{H}_{\tau}(\Sigma)$ which is flat. Let us compute the determinant of the product $c_{1} \ldots c_{m}$ in the regular representation of this algebra (which is finite dimensional if $H$ is the sphere). On the one hand, it is 1 , as $c_{1} \ldots c_{m}$ is a product of commutators. On the other hand, the eigenvalues of $c_{j}$ in this representation are $e^{2 \pi i j / n_{j}} e^{\tau_{k j}}$ with multiplicity $|\Gamma| / n_{j}$. Computing determinants as products of eigenvalues, we get a nontrivial equation on $\tau_{k j}(\hbar)$, which means that the deformation $\mathcal{H}_{\tau}$ is not flat.

Remark 3.14. In the case when $H$ is the Euclidean plane (so $\Gamma=\mathbb{Z}_{\ell} \ltimes \mathbb{Z}^{2}$, $\ell=1,2,3,4,6)$, the algebras $\mathcal{H}_{\tau}(\Sigma)$ were considered in [EOR], and it was proved (by a different method) that they are flat?. The case $\ell=1$ is trivial. In the special case when $\ell=2$, this was done earlier by Sahi, Noumi, and Stokman ([Sa], [NoSt, $[\mathbf{S t}]$ ); in this case the Hecke algebra is a generalized Cherednik's double affine Hecke algebra algebra of rank 1 (for the affine root system $C^{\vee} C_{1}$ ) that "controls" Askey-Wilson polynomials.

Remark 3.15. If $g=0$, then finite dimensional representations of $\mathcal{H}_{\tau}(\Sigma)$ are closely related to solutions of the multiplicative Deligne-Simpson problem, see [CB].

Example 3.16. This is a "multivariate" version of the previous example. Namely, letting $X, G, \Gamma$ be as in the previous example, and $n \geq 1$, we consider the variety $X^{n}$ with the action of $S_{n} \ltimes G^{n}$. Then $\mathcal{H}_{\tau}\left(X^{n}, S_{n} \ltimes G^{n}\right)$ is a flat deformation of the group algebra $\mathbb{C}\left[S_{n} \ltimes \Gamma^{n}\right]$ when $X$ is Euclidean or hyperbolic. If $n>1$, this algebra has one more essential parameter than for $n=1$; thus we can regard it as a 1-parameter deformation of $S_{n} \ltimes \mathcal{H}_{\tau^{\prime}}(X, G)$, where $\tau=\left(\tau^{\prime}, k\right)$, and $k$ is a scalar parameter (in fact, there are two additional parameters, but one of them is redundant). These algebras are considered in the paper [EGO].

Example 3.17. Recall that in EG] Ginzburg and the author attach to any finite subgroup $G$ in $S p(2 n, \mathbb{C})$ a family of algebras called symplectic reflection algebras, parametrized by a complex number $t$ and a function $c$ on the set of conjugacy classes of symplectic reflections in $G$. Here we are going to define a lattice analog of this construction, by assigning a family of algebras parametrized by functions on the set of conjugacy classes of affine reflections to any finite subgroup $G \subset S p(2 n, \mathbb{Z})$.

Let $\mathbf{L}$ be a symplectic lattice of $\operatorname{rank} 2 n$, and $G$ a finite subgroup of $S p(\mathbf{L})$. We would like to deform the group algebra of the group $\Gamma=G \ltimes \mathbf{L}$. For this purpose we

\footnotetext{
${ }^{2}$ Note that closely related flat algebras (called multiplicative preprojective algebras) were considered in [CBS.
} 
will realize $\Gamma$ as the orbifold fundamental group and then pass to the corresponding Hecke algebra. To do so, let $U=\mathbb{R} \otimes \mathbf{L}$ be the corresponding symplectic vector space. Let $\omega$ be the symplectic form on this space. To make things simple, assume that $\omega$ is a unique, up to scaling, $G$-invariant symplectic form on $U$. Pick a $G$ invariant unitary structure on $U$, i.e., a positive definite inner product $($,$) such$ that the operator $I: U \rightarrow U$ defined by

$$
\omega(v, w)=(I v, w)
$$

satisfies the equation $I^{2}=-1$. This can be done by suitably normalizing any $G$-invariant positive definite inner product on $U$ (indeed, the operator $I$ defined by (5) is then skew-adjoint under (,), so $I^{2}$ is self-adjoint, thus $I^{2}=\lambda \in \mathbb{R}$, since $\omega\left(I^{2} v, w\right)$ is another skew-symmetric invariant form on $U$; and $\lambda<0$ since $I$ has imaginary eigenvalues). This makes $U$ into a complex $n$-dimensional vector space (with complex structure defined by $I$ ), on which $G$ acts $\mathbb{C}$-linearly. Consider the compact complex torus $X=U / \mathbf{L}$. Clearly, $G$ acts holomorphically on $X$, $\pi_{1}^{\text {orb }}(X / G)=\Gamma$, and $\pi_{2}(X)=0$, so we can define a flat deformation of $\mathbb{C}[\Gamma]$, the Hecke algebra $\mathcal{H}_{\tau}(X, G)$.

We will now show that the essential parameters in this family of algebras are a complex number $q$ and a function on the set of conjugacy classes of affine reflections in $G \ltimes \mathbf{L}$, i.e., elements whose fixed set in $U$ has real codimension 2 (or complex codimension 1); this is analogous to [EG].

Indeed, the parameters $\tau$ correspond by an invertible transformation to $(c, \eta)$. Recall that $c$ is a function on the set $S$ of pairs $(Y, g)$ such that $1 \neq g \in G$ and $Y \subset X^{g}$ is a reflection hypersurface. We observe that $S$ is in a natural bijection with the set of conjugacy classes of affine reflections in $G \ltimes \mathbf{L}$. Thus it remains to observe that $\eta$ gives only one more essential parameter, since for any $Y$, the line bundle $\mathcal{O}_{X}(G Y)$ is $G$-equivariant, and thus its first Chern class is a multiple of $\omega$.

Example 3.18. (D. Kazhdan). This is a multidimensional version of Example 3.12. Let $\widetilde{X}$ be the $n$-dimensional complex hyperbolic space $\mathbf{H}_{\mathbb{C}}^{n}$, and $\Gamma$ be a finitely generated discrete group of motions of $\widetilde{X}$, i.e. a discrete subgroup of $P S U(n, 1)$. (There are a number of interesting groups of this type generated by complex hypebolic reflections, such as Mostow groups $\Gamma(p, t), \underline{\mathrm{Mo}})$. Let $\Gamma^{\prime}$ be a normal subgroup of finite index in $\Gamma$ which acts freely on $\widetilde{X}$ (it exists by Selberg's lemma), and let $X=\widetilde{X} / \Gamma^{\prime}, G=\Gamma / \Gamma^{\prime}$. Then the Hecke algebra $\mathcal{H}_{\tau}(X, G)$ is flat by Theorem 3.7 (since $\pi_{2}(X)=0$ ).

Remark 3.19. In fact, in the theory of Cherednik algebras for analytic varieties discussed in this section, it is not important that the group $G$ is finite, but it only matters that it acts on $X$ properly discontinuously. Thus, if $X$ is a complex manifold and $G$ a discrete group acting properly discontinuously and holomorphically on $X$, then one can define the Hecke algebra $\mathcal{H}_{\tau}(X, G)$ and show (similarly to Theorem 3.7) that this Hecke algebra is flat if $\pi_{2}(X) \otimes \mathbb{Q}=0$. Moreover, it is easy to see that $\mathcal{H}_{\tau}(X, G)$ is isomorphic to $\mathcal{H}_{\tau}\left(\widetilde{X}, \pi_{1}^{\text {orb }}(X / G)\right)$. (This shows, for instance, that the Hecke algebras of Example 3.18 are independent on the choice of the group $\Gamma^{\prime}$ ).

\section{A ReView OF FURTHER RESUlts}

The goal of this section is to describe the developments in the theory of Cherednik and Hecke algebras of varieties since the appearance of [Et1]. 
4.1. Representation-theoretic results and generalization of the theory of D-modules to Cherednik algebras. The methodology developed in [Et1 was used in $\mathrm{BE}$ to develop the theory of induction and restriction functors for Cherednik algebras, and in particular (in the appendix to $[\mathrm{BE}]$ ) to determine the reducibility locus of the polynomial representation of the trigonometric Cherednik algebra, giving a new proof of a result of Cherednik. Later, in BGi] Bellamy and Ginzburg generalized restriction functors of $\mathrm{BE}$ to Cherednik algebras of varieties, and introduced analogs of Kashiwara's V-filtration and specialization for these algebras.

The representation theory of $H_{1, c, \psi, X, G}$ was studied more systematically in Wi. Namely, this paper determines the possible supports of $\mathcal{O}$-coherent $H_{1, c, \psi, X, G^{-}}$ modules, and introduces the corresponding category $O_{c, \psi}(X, G)$ of modules over this algebra. This category was further studied by D. Thompson in [T1]. In particular, in this paper he showed that the KZ functor for the algebra $H_{1, c, \psi, X, G}$ is essentially surjective onto the category of finite dimensional representations of the associated Hecke algebra, generalizing a result of Losev [L1] in the linear case. This provides an analog of the Riemann-Hilbert correspondence for sheaves of Cherednik algebras. In the next paper [T2] he generalized to $H_{1, c, \psi, X, G}$ the basic setup of the theory of $G$-equivariant twisted $D$-modules (i.e., the case $c=0$ ), such as GK dimension, singular support, holonomicity, direct and inverse images, etc. This is again a generalization of the work of Losev in the linear case, [L2], and of results from $[\mathrm{BM}]$.

4.2. Deformation-theoretic results. Let $M$ be an affine symplectic variety with a symplectic action of a finite group $G$. For $g \in G$, let $M^{g} \subset M$ be the fixed set of $g$. Let $A_{+}$be a $G$-equivariant formal quantization of $M$ over $\mathbb{C}[[\hbar]]$, and $A=A_{+}\left[\hbar^{-1}\right]$. It is shown in $[\mathrm{DE}]$ that

$$
H H^{2}(A[G])=H H^{2}\left(A^{G}\right)=\left(H^{2}(M) \oplus \oplus_{g, i: \operatorname{codim} M_{i}^{g}=2} H^{0}\left(M_{i}^{g}\right)\right)^{G},
$$

where $M_{i}^{g}$ are the connected components of $M^{g}$. It is conjectured in [DE] that deformations of $A[G]$ and $A^{G}$ are unobstructed, i.e., there exists a universal deformation parametrized by this $H H^{2}$. If $M=T^{*} X$, then this follows from Theorem 2.23, so this conjecture is a generalization of Theorem 2.23 . The conjecture was proved by Halbout and Tang for $G=\mathbb{Z} / 2$ (in the similar setting of smooth manifolds), [HT].

4.3. Cherednik algebras of curves. In [FG] Finkelberg and Ginzburg studied Cherednik algebras $H_{t, c, \psi, X, G}$ attached to $X=C^{n}$ and $G=S_{n}$, where $C$ is a smooth algebraic curve (Example 2.36). In particular, they generalized to the case of an arbitrary curve the Hamiltonian reduction construction of the spherical subalgebra $\mathrm{e} H_{t, c, \psi, X, G} \mathrm{e}$, which is given in [EG] in the special case $C=\mathbb{A}^{1}$. They also studied the Hamiltonian reduction functor from the category of mirabolic character D-modules associated to $C$ to the category of representations of this spherical Cherednik algebra, generalizing the results of Gan and Ginzburg in the case of $C=\mathbb{A}^{1}$. Later McGerty and Nevins proved a derived microlocalization theorem for these algebras at spherical values of the parameter, $\underline{\mathrm{MN}}$. Also, Calogero-Moser spaces attached to this algebra (Example 2.36) arise as spaces of ideals in the algebra of differential operators on $C$ (when $C$ is affine), see $\mathrm{Be}$ and references therein. 
4.4. Cherednik algebras of abelian varieties and elliptic Calogero-Moser systems for complex crystallographic reflection groups. The Cherednik algebra attached to an abelian variety $X$ (for example, $X=E^{n}$, where $E$ is an elliptic curve) with an action of a finite group $G$ is studied in detail in [EM2, EFMV]. Namely, the paper EM2 studies a commuting family of Dunkl operators attached to $X, G$, which act not on functions but rather on sections of line bundles, i.e. they depend on the "dynamical" parameter characterizing a line bundle on $X$ (recall that for a general $X, G$ we don't have such a family at all). These operators are generalizations to the complex crystallographic reflection group case of elliptic Dunkl operators defined in 1994 by Buchshtaber, Felder, and Veselov in the case of Weyl groups, $\mathrm{BFV}$. These operators give rise to certain representations of $H_{1, c, \psi, X, G}$ which are coherent as $\mathcal{O}_{X / G}$-modules, and application of the generalized KZ functors to these representations yields monodromy representations of the Hecke algebra attached to $X, G$ (in particular, of Cherednik's DAHA in the case of Weyl groups). In [EFMV] elliptic Dunkl operators were used to prove integrability of elliptic Calogero-Moser systems for complex crystallographic reflection groups, realizing the idea of [BFV] (for Weyl groups, this had been proved by Cherednik in 1995). In the rational and trigonometric case, the quantum Hamiltonians of the Calogero-Moser system are obtained as symmetric polynomials of the Dunkl operators, but in the elliptic case this does not work literally, since Dunkl operators act on sections of line bundles rather than on functions. Still, it is shown that this approach works if instead of symmetric polynomials one takes classical Calogero-Moser Hamiltonians, in which momentum variables are the elliptic Dunkl operators and position variables are the dynamical parameters labelling the line bundle on which they act. This implies that the elliptic Calogero-Moser system can be described as the algebra of global sections of the sheaf $\mathrm{e} H_{1, c, \psi, X, G} \mathrm{e}$ for the critical value of the twist $\psi=\psi(c)$. This is similar to the description of the quantum Hitchin system in the geometric Langlands theory, as the algebra of global twisted differential operators on the moduli stack of principal bundles on a curve for a critical value of twisting. Thus, we obtain quantum integrable systems for any complex crystallographic reflection group, which are new outside the Weyl group case.

4.5. Cherednik algebras of projective spaces and projective quadrics. A classical result in the theory of D-modules says that the projective space is D-affine, i.e. the functor of global sections defines an equivalence between the category of Dmodules on a projective space and the category of modules over the algebra of global differential operators; this is a special case of the Beilinson-Bernstein localization theorem. In other words, the sheaf of differential operators on a projective space is affine. Moreover, this generalizes to the sheaf of twisted differential operators when the twisting parameter is not a negative integer. The paper [BM] generalizes this result to Cherednik algebras of the projective space, $H_{1, c, \psi, \mathbb{P}(V), G}$, where $V$ is a linear representation of a finite group $G$ (so that the classical result is recovered for $c=0)$. Namely, it shows that the sheaf $H_{1, c, \psi, \mathbb{P}(V), G}$ for generic parameters $c, \psi$ is affine, and determines explicitly the exceptional set. It is also interesting to consider the sheaf $H_{1, c, \omega, Q, G}$, if $G$ preserves a nondegenerate quadratic form $\mathbf{q}$ on $V$, and $Q \subset \mathbb{P}(V)$ is the quadric $\mathbf{q}=0$. In this case, it turns out that the algebra of global sections of $H_{1, c, \psi, Q, G}$ coincides with the central reduction of the Dunkl 
angular momenta algebra defined in [FH]. This is discussed in much more detail in FT].

4.6. Algebraic PBW theorems for Hecke algebras of complex manifolds with a finite group action. Let $X$ be a connected complex manifold and $G$ be a finite group of holomorphic transformations of $X$. Then Theorem 3.7 says that the Hecke algebra $\mathcal{H}_{\tau}(X, G)$ saisfies the formal PBW theorem, provided that $\pi_{2}(X) \otimes \mathbb{Q}=0$. However, the Hecke algebra has an algebraic version $\mathcal{H}(X, G, \mathbf{q})$ over $\mathbb{C}\left[\mathbf{q}, \mathbf{q}^{-1}\right]$, where $\mathbf{q}=\left(q_{j Y}\right), q_{j Y}=e^{\tau_{j Y}}$, and one may ask if in the same situations we have the algebraic PBW theorem: the algebra $\mathcal{H}(X, G, \mathbf{q})$ is a free module over $\mathbb{C}\left[\mathbf{q}, \mathbf{q}^{-1}\right]$. This is a much stronger statement than the formal PBW theorem given by Theorem 3.7 and it is known in very few situations (apart from the classical cases of finite, affine, and double affine Hecke algebras associated to Weyl groups). Namely, for $X$ a vector space and $W$ acting linearly, this is the Broué-Malle-Rouquier conjecture, BMR, which is now is finally proved (over $\mathbb{C}$ ), see Et2. Also, if $X$ is a compact Riemann surface of genus $\geq 1$, the algebraic PBW theorem is proved in EOR, ER1]. Finally, the paper ER2 studies the case $X=C P^{1}$, in which $\pi_{2}(X) \otimes \mathbb{Q} \neq 0$ and the PBW theorem fails (already at the formal level), and determines the flatness locus of the Hecke algebra in the space of parameters.

\section{REFERENCES}

[AFLS] J. Alev, M. Farinati, T. Lambre, A. Solotar, Homologie des invariants d'une algebre de Weyl sous l'action d'un group fini, Journal of Algebra 232, 564-577 (2000).

[BB] A. Beilinson and J. Bernstein, Proof of Jantzen's conjecture, Advances in Soviet Mathematics 16 (1993), 1-50.

[BGi] G Bellamy, V Ginzburg, Hamiltonian reduction and nearby cycles for mirabolic Dmodules, Advances in Mathematics 269, 71-161.

[BM] G. Bellamy, M. Martino, Affinity of Cherednik algebras on projective space, Algebra and Number theory, Vol. 8 (2014), No. 5, 1151-1177.

[BC] Yu. Berest and O. Chalykh, Quasi-invariants of complex reflection groups, Compos. Math. 147 (2011), no. 3, 965-1002.

[BEG] Yu. Berest, P. Etingof, V. Ginzburg, Cherednik algebras and differential operators on quasi-invariants, Duke Math. J. 118, no. 2 (2003), 279-337.

[BEG1] Yu. Berest, P. Etingof, V. Ginzburg, Finite dimensional representations of rational Cherednik algebras, Int. Math. Res. Not. 2003, no. 19, 1053-1088.

[Be] Yu. Berest, Calogero-Moser Spaces over Algebraic Curves, Selecta Mathematica 14(3), 2008.

[BE] R. Bezrukavnikov, P. Etingof, Parabolic induction and restriction functors for rational Cherednik algebras, Selecta Mathematica, New Series 14 (3), 397-425, 2009.

[Bo] A. Borel et al., Algebraic D-modules, Academic Press, Boston, 1987.

[BMR] M. Broué, G. Malle and R. Rouquier, Complex reflection groups, braid groups, Hecke algebras, J. Reine Angew. Math. 500 (1998), 127-190.

[BG] J.-L. Brylinski, E. Getzler, The homology of algebras of pseudodifferential symbols and the noncommutative residue, K-theory, v.1 (1987), p.385-403.

[BFV] V. Buchstaber, G. Felder, A. Veselov, Elliptic Dunkl operators, root systems, and functional equations. Duke Math. J., 76 (1994), p. 885-911.

[C] Cartan, H.: Quotient d'un espace analytique par un groupe d'automorphismes, Algebraic geometry and Topology (A symposium in honor of S. Lefschetz), 90-102 (1957) Princeton Univ. Press.

[Ch] I. Cherednik: Double affine Hecke algebras, Knizhnik-Zamolodchikov equations, and Macdonald operators, IMRN (Duke Math. J.) 9 (1992), p.171-180. 
[CB] W. Crawley-Boevey, Indecomposable parabolic bundles and the existence of matrices in prescribed conjugacy class closures with product equal to the identity, math.AG/0307246, Publ. Math. Inst. Hautes Etudes Sci. 100 (2004), 171-207.

[CBH] W. Crawley-Boevey, M. Holland, Noncommutative deformations of Kleinian singularities, Duke Math. J. 92 (1998), no. 3, 605-635.

[CBS] W. Crawley-Boevey, P. Shaw, Multiplicative preprojective algebras, middle convolution and the Deligne-Simpson problem, math.RA/0404186 Adv. Math. 201 (2006), 180-208.

[Da] M. Davis, Lectures on orbifolds and reflection groups, in Transformation Groups and Moduli Spaces of Curves (eds. L. Ji and S-T. Yau), ALM 16, Higher Education Press, Beijing 2010, pp. 63-93.

[dJ] A. J. de Jong et al, "Algebraic spaces", The stacks project, stacks.math.columbia.edu

[DE] V. Dolgushev, P. Etingof, Hochschild Cohomology of Quantized Symplectic Orbifolds and the Chen-Ruan Cohomology, Int. Math. Res. Not. 27 (2005) 1657-1688; ITEP-TH-43/04; math.QA/0410562

[Dr] Drinfeld, V.G., On quasitriangular Quasi-Hopf algebras and a group closely connected with $\operatorname{Gal}(\bar{Q} / Q)$, Leningrad Math. J. 2 (1991) 829-860.

[DO] C. Dunkl, E. Opdam, Dunkl operators for complex reflection groups, Proc. London Math. Soc. (3) 86 (2003), no. 1, 70-108.

[EM1] P. Etingof, X. Ma, Lecture notes on Cherednik algebras, arXiv:1001.0432

[EM2] P. Etingof, X. Ma, On elliptic Dunkl operators, Michigan Math. J. Volume 57 (2008), 293-304.

[Et1] P. Etingof, Cherednik and Hecke algebras of varieties with a finite group action, arXiv:0406499, version 3.

[Et2] P. Etingof, Proof of the Broué-Malle-Rouquier conjecture in characteristic zero (after I. Losev and I. Marin - G. Pfeiffer), arXiv:1606.08456

[ER1] P. Etingof and E. Rains, New deformations of group algebras of Coxeter groups, Int. Math. Res. Not., no. 10, pp. 635-646, 2005.

[ER2] P. Etingof and E. Rains. New deformations of group algebras of Coxeter groups. II. Geom. Funct. Anal., 17(6):1851-1871, 2008.

[EFMV] P. Etingof, G. Felder, X. Ma, A. Veselov, On elliptic Calogero-Moser systems for complex crystallographic reflection groups, J. Algebra 329, 107-129, (2011); arXiv:1003.4689

[EG] P. Etingof and V. Ginzburg, Symplectic reflection algebras, Calogero-Moser space, and deformed Harish-Chandra homomorphism, math.AG/0011114, Invent. Math., v.147(2), 2002, p.243-348.

[EG1] P. Etingof and V. Ginzburg, On m-quasi-invariants of Coxeter groups, math.QA/0106175, Mosc. Math. J., 2002, Volume 2, Number 3, Pages 555-566.

[EOR] P. Etingof, A. Oblomkov, E. Rains, Generalized double affine Hecke algebras of rank 1 and quantized Del Pezzo surfaces, math.QA/0406480 Adv. Math. 212 (2007), no. 2, 749-796.

[EGO] P. Etingof, W. L. Gan, A. Oblomkov, Generalized double affine Hecke algebras of higher rank, J. Reine Angew. Math. 600 (2006), 177-201.

[FV] M. Feigin and A. Veselov, Quasi-invariants of Coxeter groups and m-harmonic polynomails, IMRN 2002:10 (2002), 521-545.

[FH] M. Feigin, T. Hakobyan, On Dunkl angular momenta algebra, High Energ. Phys. (2015) 2015: 107. doi:10.1007/JHEP11(2015)107.

[FT] M. Feigin, D. Thompson, The global sections of the sheaf of Cherednik algebras of a smooth quadric, http://www-math.mit.edu/ dthomp/FHalgebra.pdf

[FG] M. Finkelberg, V. Ginzburg, Cherednik algebras for algebraic curves, Representation Theory of Algebraic Groups and Quantum Groups, Progr. Math., vol. 284, Birkhäuser, Springer, New York (2010), pp. 121-153.

[GGOR] V. Ginzburg, N. Guay, E. Opdam, R. Rouquier, On the category O for rational Cherednik algebras, Inventiones mathematicae, 154 (December 2003), no. 3 pp. 617-651, math.RT/0212036

[HT] G. Halbout, X. Tang, Dunkl operators and quantization of $\mathbb{Z}_{2}$-singularity, J. Reine Angew. Math., 673 (2012), pp. 209-235.

[HTT] R. Hotta, K. Takeuchi, T Tanisaki, D-Modules, Perverse Sheaves, and Representation Theory, Birkhäuser, Boston, 2008.

[L1] I. Losev, Finite-dimensional quotients of Hecke algebras, Algebra and Number Theory, Vol. 9 (2015), No. 2, 493-502. 
[L2] I. Losev, Bernstein inequality and holonomic modules (with a joint appendix with Pavel Etingof), arXiv:1501.01260, To appear in Adv. Math.

[MN] K. McGerty, T. Nevins, Derived equivalence for quantum symplectic resolutions, Selecta Math. (N.S.) 20 (2014), no. 2, 675-717.

[Mo] G. D. Mostow, On a remarkable class of polyhedra in complex hyperbolic space, Pacific J. Math. 86 (1980), no. 1, 171-276.

[NoSt] M. Noumi, J. Stokman, Askey-Wilson polynomials: an affine Hecke algebraic approach, math.QA/0001033, in Laredo Lectures on Orthogonal Polynomials and Special Functions(R. Alvarez-Nodarse, F. Marcellan and W.Van Assche, Eds), pp. 111-144, Nova Science Publishers, 2004.

[Sa] S. Sahi, Nonsymmetric Koornwinder polynomials and duality, Ann. of Math. (2) 150 (1999), no. 1, 267-282.

[St] J. Stokman, Koornwinder polynomials and affine Hecke algebras. Internat. Math. Res. Notices 2000, no. 19, 1005-1042.

[T1] D. Thompson, The image of the KZ functor for Cherednik algebras of varieties with finite group actions, arXiv: 1601.01634 .

[T2] D. Thompson, Holonomic modules over Cherednik algebras, I, arXiv:1608.01641

[Wi] S. Wilcox, Supports of representations of the rational Cherednik algebra of type A, arXiv:1012.2585

Department of Mathematics, Massachusetts Institute of Technology, Cambridge, MA 02139, USA

E-mail address: etingof@math.mit.edu 